\title{
ECONOMIC STUDIES
}

\author{
Claude Harvey
}

Sperry Research Center, Sudbury, MA 01776

\author{
SPERRY LOW TEMPERATURE GEOTHERMAL \\ CONVERSION SYSTEM - PHASE 1 \\ Contract No. DE-AC03-78ET27125 \\ (Formerly ET-78-C-02-4633) \\ and PHASE II \\ Contract No. DE-AC03-79-ET27131
}

FINAL REPORT - VOLUME VI

SRC-CR-83-43

Edited by: Warren McBee

Prepared for

U.S. Department of Energy.

San Francisco Operations Office

133 Broadway

Oaklank, CA 94612 


\section{DISCLAIMER}

This report was prepared as an account of work sponsored by an agency of the United States Government. Neither the United States Government nor any agency Thereof, nor any of their employees, makes any warranty, express or implied, or assumes any legal liability or responsibility for the accuracy, completeness, or usefulness of any information, apparatus, product, or process disclosed, or represents that its use would not infringe privately owned rights. Reference herein to any specific commercial product, process, or service by trade name, trademark, manufacturer, or otherwise does not necessarily constitute or imply its endorsement, recommendation, or favoring by the United States Government or any agency thereof. The views and opinions of authors expressed herein do not necessarily state or reflect those of the United States Government or any agency thereof. 


\section{DISCLAIMER}

Portions of this document may be illegible in electronic image products. Images are produced from the best available original document. 


\section{DISCLAIMER}

This report was prepared as an account of work sponsored by an agency of the United States Government. Neither the United States Government nor any of their emp loyees, makes any warranty, express or implied, or assumes any legal liability or responsibility for the accuracy, completeness, or usefulness of any information, apparatus, product, or process disclosed, or represents that its use would not infringe privately owned rights. Reference herein to any specific commercial product, process, or service by trade name, trademark, manufacturer, or otherwise, does not necessarily constitute or imply its endorsement, recommendation, or favoring by the United States Government or any agency thereof. The views and opinions of authors expressed here in do not necessarily state or reflect those of the United States Government or any agency thereof. 


\section{FOREWORD}

This final report covers the work performed under the "Sperry Low Temperature Geothermal Conversion System" project, both Phase I under Contract No. DE-AC03-78-ET27125 (formerly ET-78-C-02-4633) and Phase II under Contract No. DE-AC03-79-ET27131.

Vols. I and II, submitted in 1982 covered a portion of the work accomplished under Phase I. Vols. III, IV, V, and VI submitted at this time cover the balance of work performed under Phase I and work performed under Phase II. 
VOLOME TABLE OF CONTENTS

I ORGANIC WORKING FLOID PROPERTIES

II SYSTEM COMPONENT COMPATIBILITI \& R-114 STABILITY

III SYSTEMS DESCRIPTION

IV FIETD ACTIVITIES

V COMPONENT DEVELOPMENT

VI ECONOMIC STUDIES 
VOLUME VI

TABLE OF CONTENTS

6.0

INTRODUCTION

1

6.1

ECONOMIC STUDY OF GRAVITY HEAD

3

VS. CONVENTIONAL BINARY PLANTS

- PART I

6.1 .1

6.1 .2

Scope, Menthodology \& Bases

Results of Sensitivity Study

$\frac{3}{6}$

6.2

ECONOMIC STUDY OF GRAVTIY HEAD

8

VS. CONVENTIONAL BINARY PLANTS

- PART II

6.2 .1

Scope, Menthodology \& Bases

8

6.2 .2

Results of Part II Study

10

6.3

PLANT PERFORMANCE CALCULATIONS

11

$6 \cdot 3 \cdot 1$

Assumptions and Conditions

11

$6 \cdot 3 \cdot 1 \cdot 1$

Condensing Conditions

Fluid Properties

11

12

6.3 .2

Conventional Binary Plants

Using RI14

$6.3 \cdot 2.1$

RII 4 Parameters

12

$6.3 \cdot 2.2$

$6 \cdot 3 \cdot 2 \cdot 3$

$6.3 \cdot 2.4$

$6.3 \cdot 2 \cdot 5$

$6.3 \cdot 2.6$

$6 \cdot 3 \cdot 2 \cdot 7$

Generator Output

Feed Pump

Brine Pump Power

Condenser Load

Busbar Output

Temperature Rise Due to Feed Pumping

$6 \cdot 3 \cdot 3$

$6.3 \cdot 3.1$

$6 \cdot 3 \cdot 3 \cdot 2$

$6 \cdot 3 \cdot 3 \cdot 3$

$6 \cdot 3 \cdot 3 \cdot 4$

$6 \cdot 3 \cdot 3 \cdot 5$

$6 \cdot 3 \cdot 3.6$

$6 \cdot 3 \cdot 3 \cdot 7$

Gravity Head Plent $\quad 16$

RI14 Conditions $\quad 16$

Downhole Brine Pump $\quad 16$

Lift Work from 2,280 ft. $\quad 17$

Ideal Energy Split Between 18

Pelton Wheel \& Main Turbine

Generator Output

18

Condenser Parasitic Loed $\quad 19$

Busbar Power

19

Conventional Binary Plant $\quad 19$

$6.3 \cdot 4.1$

6.3 .4 .2

Using Isobutane at 900 PSI

Organic Parameters

19

Generator Output 19

Feed Pump Work 20

Brine Pump Power 20

Condenser Parasitic Load 20

Busbar Output 20

Temperature Rise Due to 21

Feed Pumping 
TABLE OF CONTENTS (Cont'd)

6.3 .5

$$
\begin{aligned}
& 6 \cdot 3 \cdot 5 \cdot 1 \\
& 6 \cdot 3 \cdot 5 \cdot 2 \\
& 6 \cdot 3 \cdot 5 \cdot 3 \\
& 6 \cdot 3 \cdot 5 \cdot 4 \\
& 6 \cdot 3 \cdot 5 \cdot 5 \\
& 6 \cdot 3 \cdot 5 \cdot 6 \\
& 6 \cdot 3 \cdot 5 \cdot 7
\end{aligned}
$$

6.3 .6

$$
\begin{aligned}
& 6 \cdot 3 \cdot 6.1 \\
& 6 \cdot 3 \cdot 6.2 \\
& 6 \cdot 3 \cdot 6.3 \\
& 6.3 \cdot 6.4
\end{aligned}
$$

6.4

CONCLUSIONS

$$
\text { Conventional Binary Plant }
$$$$
\text { Using RII4 at } 900 \text { PSI }
$$

RI14 Parameters

Generator Output

Feed Pump Work

Brine Pump Power

Condenser Lood

Busber Output

Temperature Rise Due to Feed Pumpting

Brine Pumping Requirements Assumptions \& Conditions Conventional Binary Plant Gravity Head Plant

Necessary Pump Setting Depths

21

21

22

22

22

23

23

23

23

24

25

26

28 


\section{LIST OF ILLUSTRATIONS}

Figure

Page

1

Effect of Avoided Energy Cost on Binary and G.H. ROI

29

2 Fffect of Capital Cost on Binary and G.H. ROI

30

Effect of $O$ \& $M$ Cost on Binery and G.H. ROI

31

4

Effect of Brine Cost on Binary and G.H. ROI

32

5

Effect of Brine Cost on Five-Unit Binary \& G.H. ROI

33

6

Effect of Brine Cost on ROI, Plants Without Wells

34 


\subsection{INTRODUCTION}

At the time that the analyses contained in this volume were performed, the avoided energy costs of California's regulated electric utilities were at historic highs and were projected to continue to increase at double-digit annual rates. Since that time, oil prices have slipped, the general rate of inflation has abated, domestic electric power consumption has fallen short of expectations; and a host of other economic elements have shifted in directions which have resulted in substantial reductions in current California avoided energy costs. The question of whether these changes are merely short-term aberrations or represent a fundamental shift in the long-term energy supply and demand balance is beyond the scope of this report.

Although the present depressed avoided electric energy prices are not consistent with the projections on which this report's analyses are based, a fact which results in significant discrepancies between the absolute financial returns predicted and those achievable under current circumstances, the comparative results of the study remain valid. The basic thrust of the studies represented in this report was to compare the overall economics of the Gravity Head system with that of optimized conventional binary generating plants.

The study is site-specific to the East Mesa geothermal reservoir. It begins with an analysis of optimum individual well production based on the known characteristics of the reservoir. The limiting factors at East Mesa are reservoir drawdown and maximum practical brine pump impeller setting depth. The individual-well production limit is achieved when brine pressure at the pump inlet is reduced to the point at which $\mathrm{CO}_{2}$ starts to come out of solution, and fouling and impeller cavitation become imminent. The optimum pumping rate was found to be $1300 \mathrm{gpm}$ (at $360^{\circ} \mathrm{F}$ density) and required that the Gravity Head pump impeller be set 2,033 feet below grade. The required conventional plant pump setting was 1,887 feet below grade, 
a depth which is probably at the practical limit for shaft-driven conventional pumps.

Using the $1300 \mathrm{gpm}, 360^{\circ} \mathrm{F}$ resource as a starting point, detailed cost estimates and performance calculations were made for the Gravity Head and various conventional alternative well-head systems. The most cost effective of the conventional alternatives was found to be a 700 psi cycle using R114 as the working fluid (actually optimized at $682 \mathrm{psi}$ ). That system produced $3,805 \mathrm{~kW}$ busbar net vs. 4,995 kW busbar net for the Gravity Head unit. Since both the Gravity head and conventional binary plant cost estimates for commercially mature units came out roughly equal, it was obvious that the Gravity Head system would produce more attractive financial returns - it became a question of degree.

The study is in two parts. In Part $I$, emphasis is placed on the economic sensitivities of the compared systems to variations in capital cost, O\&M cost, avoided energy inflation rates, and brine cost. In Part II, a somewhat more rigorous attempt is made to derive the actual economic performance of the systems under more realistic circumstances with contingencies, cost penalties, and multiple-unit developments added.

The results of the Part I study are given in section 6.1. The results of the Part II study are given in section 6.2. Detailed cash flow analysis charts for both studies are presented in Appendix A. 
6.1 ECONOMIC STUDY OF GRAVITY HEAD VS. CONVENTIONAL BINARY PLANTS - PART I

\subsubsection{Scope, Methodology and Bases}

\subsubsection{Plants Compared}

- Gravity Head well-head plant using Rll4.

- Conventional binary well-head plant using Rl14 at 700 psi.

- Conventional binary well-head plant using Rl14 at 900 psi.

- Conventional binary well-head plant using isobutane at 900 psi.

\subsubsection{Methodology}

A detailed cost estimate was prepared for each of the above plants. An analysis was made of each plant cycle in order to calculate the net plant output. The most economically attractive of the conventional binary plants was selected, and a ten-year cash flow analysis was prepared. An identical analysis was also prepared for the Gravity Head plant. Return on investment sensitivity studies were "what effect does a $\$ 500,000$ variation in plant construction cost have on the ten-year R.O.I.?"

\subsubsection{Basis for Fabrication and Construction Cost-Estimates}

- Each plant was assumed to be a standardized (tenth generation) production model representing very little engineering and design cost.

- Conventional plant costs include everything required except the production well, reinjection well, downhole brine pump, brine piping to and from the wells, and electrical transmission lines.

- Gravity Head plant costs include everything required except the production well, reinjection well, spent brine piping to the injection well, and electrical transmission lines. Downhole pump cost is included.

- Costs include no mark-up profit for any of the contractors. 


\subsubsection{Brine Cost Basis}

- It is assumed that the resource holder drills and completes the production and injection wells in all cases.

- The resource holder provides the downhole pump for the conventional system.

- The plant owner provides the downhole pump for the Gravity Head system (included in plant cost).

\subsubsection{Electrical Sale Price Basis}

- Assume price per kilowatt-hour equal to 908 of San Diego Gas and Electric Company's 1981 non-time-of-day average avoided cost escalated at the annual rate reflected in S.D.G. \& E's five-year projections as published by the California P.U.C. in March 1981.

- Assume capacity price offers in effect for S.D.G. \& E as published in the March, 1981 P.U.C. publication. Capacity price payments to begin after plant shakedown.

- Plant shakedown lasts three months.

- Plant availability is 508 during shakedown and 808 thereafter.

6.1.1.6 Basis for Operating and Maintenance (O\&M) Costs

- Eight men $\times \$ 30 / \operatorname{man}-\mathrm{hr} . \times 2,080 \mathrm{hrs} . /$ year $=\$ 500 \mathrm{~K}(\mathrm{Jan} .1981$ dollars).

- Materials, crafts, and services $=\$ 500 \mathrm{~K}$ (Jan. 1981 dollars).

(The O\&M costs as a function of capital costs are excessive in this case; in actuality they would be lowered on a per plant basis by spreading them over a multi-unit complex of five units or more to be run by the same eight operators).

\subsubsection{Basis for Cash Flow Analysis}

- Financial structure is a limited partnership.

- The partners are assumed to have sufficient outside income to be able to utilize tax credits and write-offs as they occur (508 tax bracket assumed). 
- A 108 investment tax credit plus 158 alternate energy and geothermal tax credits are taken as funds are expended.

- Plant is depreciated on a five-year schedule (15-year life assumed) beginning at plant start-up per the following schedule:

\begin{tabular}{|c|c|c|c|c|c|}
\hline Plant Start-up Date & $\begin{array}{l}\text { lst } \\
\text { Year } \\
\end{array}$ & $\begin{array}{l}\text { 2nd } \\
\text { Year }\end{array}$ & $\begin{array}{l}3 \text { rd } \\
\text { Year }\end{array}$ & $\begin{array}{l}\text { 4th } \\
\text { Year }\end{array}$ & $\begin{array}{l}5 \text { th } \\
\text { Year }\end{array}$ \\
\hline $1982,83,84$ & 158 & 228 & 218 & 218 & 218 \\
\hline 1985 & 18 & 33 & 25 & 16 & 8 \\
\hline after 1985 & 20 & 32 & 24 & 16 & 8 \\
\hline
\end{tabular}

- Annual rate of inflation is assumed to be 108 calculated at $(1.1)^{\mathrm{N}}$.

- The construction period is six months (production model plants being produced in volume). Construction start date is January 1, 1982 .

- Return on investment is calculated using the Discounted Cash Flow method:

$$
\sum_{\text {year }=1}^{\text {year }=10} \frac{\text { Net cash flow for year }}{(1+i)^{\text {year }}}=0
$$

For computer use, the zero condition was assumed to be satisfied when $\sum \leq 10$.

- S.D.G. \& E avoided cost and capacity payment projections were approximated as follows:

$$
A \times(1+i)^{N}
$$

where

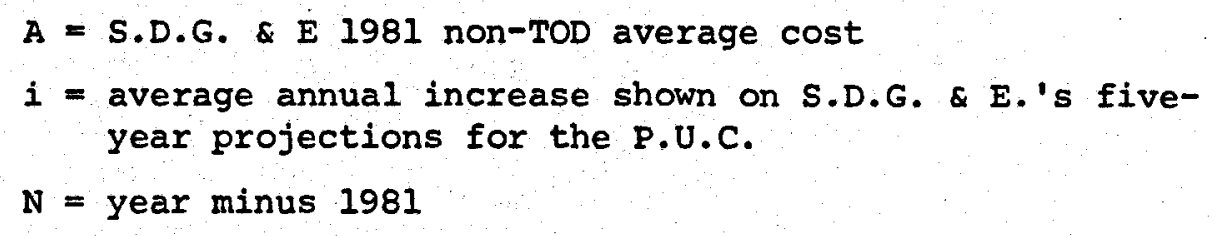


- Operating and capital costs were escalated at the assumed general rate of inflation using a January 1981 dollar basis.

- Brine costs were escalated at a rate of $65 \%$ of the assumed general rate of inflation.

\section{1 .2 Results of Sensitivity Study}

A baseline cash flow analysis was made for both a $3,800 \mathrm{~kW}$ net, 700 psi, Rll4 conventional binary system and a 5,000 kW net Gravity Head system. The baseline parameters were:

- capital cost - $\$ 5$ million

- brine cost - 30.2873\%/1000 $\mathrm{lb}$.

- O\&M cost = \$1 million/year

- general inflation rate - $108 /$ year

- utility elec. rate inflation - 118/year.

Subsequently, cash flow analyses were made while holding four of the above five parameters constant and varying the fifth with the following results.

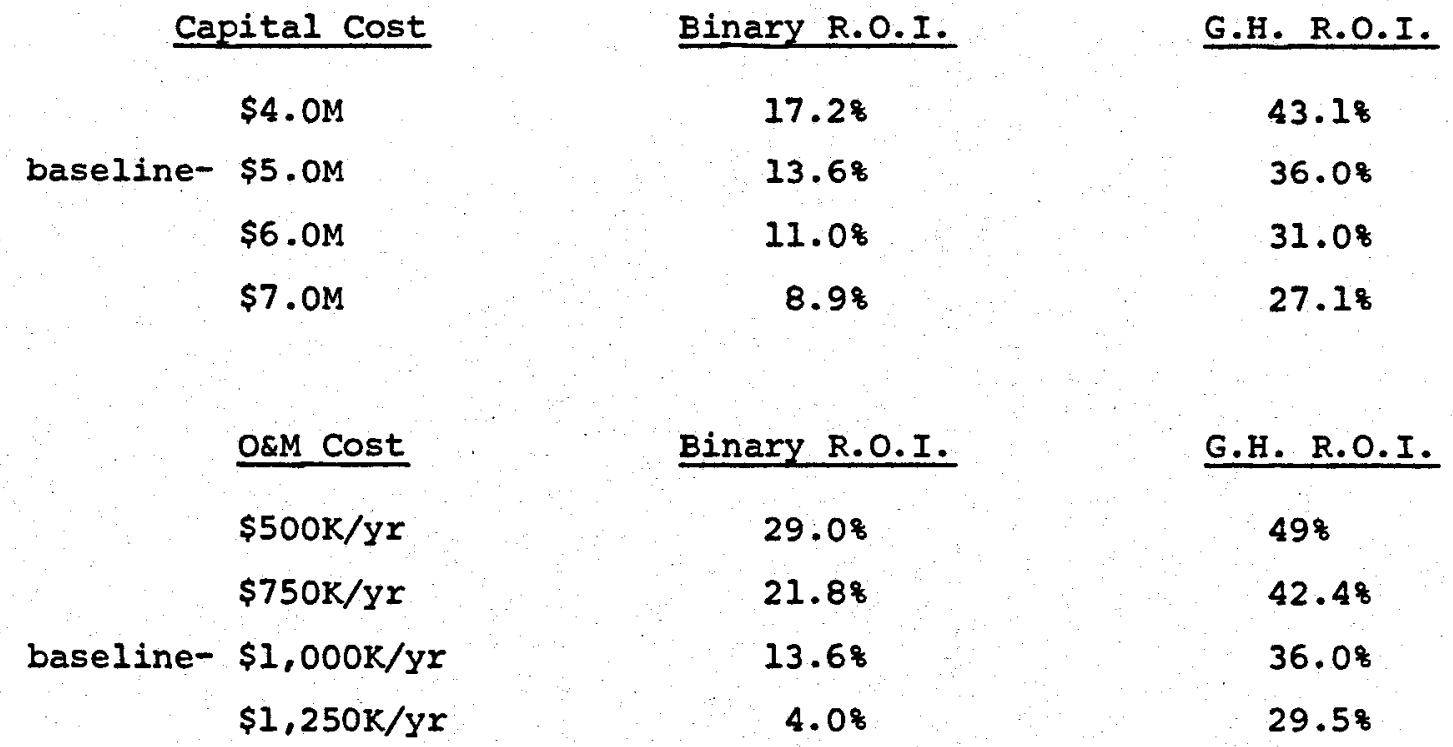




\begin{tabular}{ccc}
$\begin{array}{c}\text { Elec. Rate } \\
\text { Inflation }\end{array}$ & Binary R.O.I. & G.H. R.O.I. \\
\hline 108 & 8.48 & 32.18 \\
baseline- 118 & 13.68 & 36.08 \\
128 & 18.18 & 39.78 \\
138 & 22.28 & 43.48 \\
Brine Cost/1000 1b. Binary R.O.I. & 238 & \\
$21.6338 \mathrm{c}$ & 23.68 & G.H. R.O.I. \\
$30.2873 \mathrm{c}$ & 8.48 & 44.08 \\
$34.6141 \mathrm{c}$ & 2.38 & 36.08 \\
$38.9408 \mathrm{C}$ & & 32.08
\end{tabular}

These results are plotted in Figures $1-4$. 


\subsubsection{Scope, Methodology and Bases}

\subsubsection{Plants Compared}

- Gravity Head well-head plant using Rll4.

- Conventional binary well-head plant using Rll4 at 700 psi (the most economically attractive of the conventional alternatives studied).

\subsubsection{Methodology}

- A 108 contingency is added to the estimated capital cost of the plants. Cash flow analyses are made for single-unit Gravity Head and conventional binary plants, both with and without wells included at various assumed brine charges. Also, fiveunit cluster plants of both the Gravity Head and conventional binary type are analyzed.

\subsubsection{Basis for Fabrication and Construction Cost Estimates}

- Each plant is assumed to be a standardized (tenth generation) production model representing very little engineering and design cost.

- Conventional plant costs "without wells" include everything except the production well, reinjection well, downhole brine pump, brine piping to and from the wells, and transmission lines.

- Conventional and Gravity Head plant costs "including wells" include everything except transmission lines.

- Gravity Head plant costs "without wells" include everything required except the production well, reinjection well, spent brine piping, and electrical transmission lines. Downhole pump cost is included. A $\$ 352,000$ penalty is added to the capital cost in this case to compensate the resource holder for the additional well investment required to accommodate the Gravity Head unit (a large-bore production well vs. a conventional well plus brine pump). 
- A 108 contingency is added to all estimated costs.

- Costs include no mark-up or profit for any of the contractors.

\subsubsection{Brine Cost Basis}

- For plants "without wells" it is assumed that the resource holder drills and completes the production and injection wells, in which event:

- The resource holder provides the downhole brine pump for the conventional system.

- The plant owner provides the downhole brine pump for the Gravity Head system and pays the resource holder a one-time charge of $\$ 352,000$ to compensate him for the increased well cost. This amount is included in the Gravity Head capital cost.

- For plants with "wells included" the brine is purchased "in situ" and all well costs are borne by the plant owner.

\subsubsection{Electrical Sale Price Basis}

- Same as PART I plus the following:

- Each well-head unit shakedown lasts three months.

- Unit availability is 508 during shakedown and $80 \%$ thereafter.

- Multi-unit complexes are constructed on a staggered unit schedule.

6.2.1.6 Basis for Operating and Maintenance costs

- Single unit O\&M $=\$ 1,000 \mathrm{~K} /$ year $(\mathrm{Jan} .1981$ dollars).

- For each unit added to the first to make up a multi-unit complex, add $\$ 366 \mathrm{~K}$ (Jan. 1981 dollars).

\subsubsection{Basis for Cash Flow Analysis}

- Same as PART I. 


\subsubsection{Results of PART II Study}

\begin{tabular}{lll} 
& $\begin{array}{l}\text { Brine Cost } \\
\$ / 1000 \mathrm{lb} .\end{array}$ & $\begin{array}{l}10 \text { Year } \\
\text { R.O.I. }\end{array}$ \\
\hline Five-Unit Gravity Head & 8.6535 & 43.238 \\
Five-Unit Binary & 8.6535 & 31.348 \\
Five-Unit Gravity Head & 17.247 & 38.958 \\
Five-Unit Binary & 17.247 & 26.658 \\
& & \\
& & \\
& Brine Cost & 10 Year \\
& $\$ / 1000 \mathrm{lb}$. & R.0.I. \\
\hline Single-Unit Gravity Head & 21.6338 & 36.48 \\
Single-Unit Binary & 21.6338 & 18.38 \\
Single-Unit Gravity Head & 30.2873 & 30.08 \\
Single-Unit Binary & 30.2873 & 10.28 \\
Single-Unit Gravity Head & 38.9408 & 23.158 \\
Single-Unit Binary & 38.9408 & 0.208
\end{tabular}

These results are plotted in Figures 5 and 6. 


\subsection{PLANT PERFORMANCE CALCULATIONS}

\subsubsection{Assumptions and Conditions \\ 6.3.1.1 Condensing Conditions}

- All plants that have a $90^{\circ} \mathrm{F}$ rated condensing temperature have a $5 \%$ subcooling condition or a liquid temperature out of the condenser of $85^{\circ} \mathrm{F}$. Both temperature conditions are at a pressure of 39.0 PSIA.

- There is a 3 psi loss between turbine outlet and condenser outlet. This results in a 42.0 PSIA turbine outlet.

- In the gravity-head system, the condenser outlet conditions of a subcooled $85^{\circ} \mathrm{F}$ and 39.0 PSIA are increased in the ERU to a saturated $94^{\circ} \mathrm{F}$ and 41.75 PSIA. Although this does result in approximately $9^{\circ} \mathrm{F}$ less cooling of the brine, it does not appreciably increase the output of the system. It does, however, reduce the amount of heat exchanger and condenser surface required.

The ERU also serves the need for an RI14 storage vessel during plant standby, a distribution manifold for turbine exhaust, and a vapor desuperheater. In the case of a stand-alone turbine pump system, the restoration of temperature end pressure by the ERU does significantly decrease the pump parasitic load since less temperature is removed from the brine for the pumping function.

- One Baltimore Alr Coil unit is rated at a heat rejection capacity of $6 \times 10^{6} \mathrm{Btu} / \mathrm{hr}$ in operating between a wet bulb temperature of $60^{\circ} \mathrm{F}$ and a condensing temperature of $90^{\circ} \mathrm{F}$. The parasitic load for one unit is $21 \mathrm{hp}$ composed of two $3 \mathrm{hp}$ pumps and two $7.5 \mathrm{hp}$ fans. For consistency, the calculations treat 
the condenser load as a continuous function rather than incrementally.

\subsubsection{Fluid Properties}

- Brine thermodynamic properties from Keenan and Keys.

- Brine transport properties are from ASHRAE.

- Rl14 thermodynamic properties are from Hules (Vol. 1, this Final Report).

- Rl14 transport properties above the dome are from the several Russian sources as presented in the Sperry report SRC-CR-81-74. Subcritical properties are from ASHRAE.

- Isobutane thermodynamic properties are from the starling PH diagram of 1973.

\subsubsection{Conventional Binary Plants Using Rl14}

\subsubsection{R114 Parameters}

- Peak Rl14 temperature $=340^{\circ} \mathrm{F}$ :

$$
\begin{aligned}
& \left(\dot{w}_{B}\right) \quad(R) \\
& \dot{w}_{0}(666)=160.5 \times 2.6118=419.2 \mathrm{lb} \mathrm{sec} \\
& \dot{w}_{0}(682)=160.5 \times 2.7658=443.91 \mathrm{~b} \mathrm{sec} \\
& \dot{w}_{0}(700)=160.5 \times 2.91623=468.1 \mathrm{lb} \mathrm{sec}
\end{aligned}
$$

- Isentropic drop to 42 PSI:

$$
\begin{aligned}
& \mathrm{H}^{-}(666)=\frac{105.84 \mathrm{Btu}-90.2 \mathrm{Btu}}{1 \mathrm{~b}}=15.57 \mathrm{Btu} / \mathrm{lb} \\
& \mathrm{H}^{-}(682)=\frac{104.6 \mathrm{Btu}-89.28 \mathrm{Btu}}{1 \mathrm{~b}}=15.32 \mathrm{Btu} / 1 \mathrm{~b} \\
& \mathrm{H}(700)=\frac{103.2 \mathrm{Btu}-88.2 \mathrm{Btu}}{1 \mathrm{~b}}=15.00 \mathrm{Btu} / 1 \mathrm{~b}
\end{aligned}
$$




\subsubsection{Generator Output}

$$
\begin{aligned}
\text { Power }(666) & =\frac{15.57 \mathrm{Btu} / \mathrm{lb} \times 419.2 \mathrm{lbs} / \mathrm{sec} \times .85 \text { Eff. Turb. } \times .96 \text { Eff. Gen. }}{.9482 \mathrm{But} / \mathrm{kW}-\mathrm{sec}} \\
& =5617 \mathrm{~kW} . \\
\text { Power }(682) & =\frac{15.32 \mathrm{Btu} / \mathrm{lb} \times 443.9 \mathrm{lb} / \mathrm{sec} \times .85 \mathrm{Eff} \text {. Turb. } \times .96 \mathrm{Eff.} \mathrm{Gen.}}{.9482 \mathrm{Btu} / \mathrm{kW}-\mathrm{sec}} \\
& =5852 \mathrm{~kW} . \\
\text { Power }(700) & =\frac{15.00 \mathrm{Btu} / \mathrm{lb} \times 468.1 \mathrm{lb} / \mathrm{sec} \times .83 \mathrm{Eff.Turb.} \times 96 \mathrm{Eff.} \mathrm{Gen.}}{.9482 \mathrm{Btu} / \mathrm{kW}-\mathrm{sec}} \\
& =5901 \mathrm{~kW} .
\end{aligned}
$$

\subsubsection{Feed Pump}

- Organic volume flow at an average density of 86.88:

$$
\begin{aligned}
& Q(666)=\frac{419.2 \mathrm{lb} / \mathrm{sec} \times 60 \mathrm{sec} / \mathrm{min} \times 7.48 \mathrm{gal} / \mathrm{ft}^{3}}{86.88 \mathrm{lb} / \mathrm{ft}^{3}}=2165 \mathrm{gpm} . \\
& Q(682)=\frac{443.9 \mathrm{lb} / \mathrm{sec} \times 60 \mathrm{sec} / \mathrm{min} \times 7.48 \mathrm{gal} \mathrm{\textrm {ft } ^ { 3 }}}{86.88 \mathrm{lb} / \mathrm{ft}^{3}}=2222 \mathrm{gpm} . \\
& Q(700)=\frac{468.1 \mathrm{lb} / \mathrm{sec} \times 60 \mathrm{sec} / \mathrm{min} \times 7.48 \mathrm{gal} / \mathrm{ft}^{3}}{86.88 \mathrm{lb} / \mathrm{ft}^{3}}=2328 \mathrm{gpm} .
\end{aligned}
$$

- Electric power into feed pump:

$$
\begin{aligned}
\text { Power }(666) & =\frac{2165 \mathrm{gpm} \times(686-39) \mathrm{psi} \times 1.01 \text { (Compressibility) }}{2298 \mathrm{gpm} \times \mathrm{psi} / \mathrm{kW} \times .77 \text { (Eff. Turb.) } \times .93 \text { (Eff. Motor) }} \\
& =870 \mathrm{~kW} . \\
\text { Power }(682) & =\frac{2222 \mathrm{gpm} \times(702-39) \mathrm{psi} \times 1.01 \text { (Compressibility) }}{2298 \mathrm{gpm} \times \mathrm{psi} / \mathrm{kW} \times .77 \text { (Eff. Turb.) } \times-93 \text { (Eff. Motor) }} \\
& =904 \mathrm{~kW} . \\
\text { Power }(700) & =\frac{2342 \mathrm{gpm} \times(720-39) \mathrm{psi} \times 1.01 \text { (Compressibility) }}{2298 \mathrm{gpm} \times \mathrm{psi} / \mathrm{kW} \times .77 \text { (Eff. Turb.) } \times .93 \text { (Eff. Motor) }} \\
& =979 \mathrm{~kW} .
\end{aligned}
$$


NOTE: Compressibility is approximately 28 by diagram. Half of this should be charged to pumping energy.

6.3.2.4 Brine Pump Power

Power $(\mathrm{BP})=\frac{755 \mathrm{psi} \times 1300 \mathrm{gpm}}{2298 \mathrm{gpm} \times \mathrm{psi} / \mathrm{kW} \times .51 \text { (Eff. Pump) }}=837 \mathrm{~kW}$.

6.3.2.5 Condenser Load

- Turbine exhaust enthalpy:

$H(666)=\frac{90.27 \mathrm{Btu}+(15.57 \mathrm{Btu} \times .15 \mathrm{Eff} \text {. Loss })}{1 \mathrm{~b}}=92.61 \mathrm{Btu} / \mathrm{lb}$

$H(682)=\frac{89.28 \mathrm{BTU}+(15.32 \mathrm{Btu} \times .15 \mathrm{Eff} \text { Loss })}{1 \mathrm{~b}}=91.58 \mathrm{Btu} / \mathrm{lb}$

$H(700)=\frac{88.20 \mathrm{Btu}+(15.00 \mathrm{Btu} \times .15 \mathrm{Eff} \text {. Loss })}{1 \mathrm{~b}}=90.48 \mathrm{Btu} / 1 \mathrm{~b}$.

- Heat load in Btu/hr:

$H(666)=(92.61=29.3) \mathrm{Btu} / \mathrm{Ib}(419.2 \mathrm{lb} / \mathrm{sec} \times 3600 \mathrm{sec} / \mathrm{hr})$

$=95.5 \times 10^{6} \mathrm{Btu} / \mathrm{hr}$

$H(682)=(91.58-29.3) \mathrm{Btu} / \mathrm{lb}(443.9 \mathrm{lb} / \mathrm{sec} \times 3600 \mathrm{sec} / \mathrm{hr})$

$=99.5 \times 10^{6} \mathrm{Btu} / \mathrm{hr}$

$\mathrm{H}(700)=(90.48-29.3) \mathrm{Btu} / \mathrm{Ib}(465.2 \mathrm{lb} / \mathrm{sec} \times 3600 \mathrm{sec} / \mathrm{hr})$

$=103.1 \times 10^{6} \mathrm{Btu} / \mathrm{hr}$ 
- Condenser load:

Power $(666)=\frac{95.5 \mathrm{Btu} / \mathrm{hr} \times 10^{6} \times 21.0 \mathrm{hp} \times .746 \mathrm{~kW} / \mathrm{hp}}{6 \times 10^{6} \mathrm{Btu} / \mathrm{hr} \times .85 \mathrm{Eff.} \mathrm{Motor}}=293 \mathrm{~kW}$ Power (682) $=\frac{99.5 \mathrm{Btu} / \mathrm{hr} \times 10^{6} \times 21.0 \mathrm{hp} \times .746 \mathrm{~kW} / \mathrm{hp}}{6 \times 10^{6} \mathrm{Btu} / \mathrm{hr} \times .85 \mathrm{Eff} . \text { Motor }}=306 \mathrm{~kW}$ Power $(700)=\frac{103.1 \mathrm{Btu} / \mathrm{hr} \times 10^{6} \times 21.0 \mathrm{hp} \times .746 \mathrm{~kW} / \mathrm{hp}}{6 \times 10^{6} \mathrm{Btu} / \mathrm{hr} \times .85 \mathrm{Eff} \text {. Motor }}=318 \mathrm{~kW}$

\subsubsection{Busbar Output}

$$
\begin{aligned}
& \text { Power }(666)=5617 \mathrm{~kW}-870 \mathrm{~kW}-837 \mathrm{~kW}-293 \mathrm{~kW}=3617 \mathrm{~kW} . \\
& \text { Power }(682)=5852 \mathrm{~kW}-904 \mathrm{~kW}-837 \mathrm{~kW}-306 \mathrm{~kW}=3805 \mathrm{~kW} . \\
& \text { Power }(700)=5901 \mathrm{~kW}-979 \mathrm{~kW}-837 \mathrm{~kW}-318 \mathrm{~kW}=3767 \mathrm{~kW} .
\end{aligned}
$$

\subsubsection{Temperature Rise Due to Feed Pumping (682 psi)}

- Reversible increase:

$\Delta \mathrm{T}=3.5^{\circ} \mathrm{F}$ (from diagram)

- Temperature increase due to impeller loss:

$\Delta \mathrm{H}=979 \mathrm{~kW} \times .93$ (Eff.) $\times .2$ (Eff. Loss) $=182 \mathrm{~kW}$.

$$
\Delta \mathrm{H}_{1 \mathrm{~b}}=\frac{182 \mathrm{~kW} \times .9482 \mathrm{Btu} \mathrm{sec} / \mathrm{kW}}{443.9 \mathrm{lb} / \mathrm{sec}}=.39 \mathrm{Btu} / \mathrm{Lb}
$$

$\Delta T=.39 \mathrm{Btu} / \mathrm{Lb} / .22(\mathrm{Cp})=1.77^{\circ} \mathrm{F}$.

Total temperature increase $=3.5^{\circ} \mathrm{F}+1.77^{\circ} \mathrm{F}=5.27^{\circ} \mathrm{F}$.

- Temperature into heat exchanger:

$T=85^{\circ} \mathrm{F}+5^{\circ} \mathrm{F}=90^{\circ} \mathrm{F}$. 


\subsubsection{Gravity Head Plant}

\subsubsection{RI14 Conditions}

- Flow $w_{0}=160.5\left(w_{B}\right) \times 3.99015(R)=640.41 \mathrm{~b} / \mathrm{sec}$

- Conditions out of heat exchanger:

$$
\mathrm{H}=97.48 \mathrm{Btu} / \mathrm{lb} \quad \mathrm{T}=340.0^{\circ} \mathrm{F} \quad \mathrm{P}=900 \mathrm{psi}
$$

- Isentropic arop to $42 \mathrm{psi}$

- $\Delta \mathrm{H}^{\prime}=97.48 \mathrm{Btu} / \mathrm{lb}-83.43 \mathrm{Btu} / \mathrm{lb}-14.05 \mathrm{Btu} / \mathrm{lb}$ (for reference only)

\subsubsection{Downhole Brine Pump}

- Pressure added $=767 \mathrm{psi}$

- Power into impeller $\frac{1300 \mathrm{gpm} \times 767 \mathrm{psi}}{2298 \mathrm{gpm} \times \mathrm{psi} / \mathrm{kW} \times .75 \text { (Eff. Imp.) }}=578.5 \mathrm{~kW}$

- Energy rate into impeller

$578.5 \mathrm{~kW} / .9482(\mathrm{~kW}-\mathrm{sec}) / \mathrm{Btu}=548.4 \mathrm{Btu} / \mathrm{sec}$

- Energy/1b of R114

$548.4 \mathrm{Btu} / \mathrm{sec} / 640.4 \mathrm{lb} / \mathrm{sec}=.8563 \mathrm{Btu} / \mathrm{lb}$

- Isentropic energy drop across turbine $\frac{548.4 \mathrm{Btu} / \mathrm{sec}}{.75(\text { Eff. Turb. })}=731.2 \mathrm{Btu} / \mathrm{sec}$

- Energy/lb of R114 across turbine:

$$
\Delta \mathrm{H}^{-}=731.2 \mathrm{Btu} / \mathrm{sec} / 640.4 \mathrm{lb} / \mathrm{sec}=1.142 \mathrm{Btu} / 1 \mathrm{~b}
$$


- Irreversibility

$1.145 \mathrm{Btu}-.8563 \mathrm{Btu}=.2854 \mathrm{Btu}$

- Diagram $1.142 \mathrm{Btu} / \mathrm{lb}$ as isentropic drop from 97.48 Btu:

$\mathrm{H}=97.48 \mathrm{Btu} / \mathrm{Ib}-1.142 \mathrm{Btu} / \mathrm{Ib}=96.34 \mathrm{Btu} / 1 \mathrm{~b}$

$P=583$ psi by diagram

- Diagram irreversibility at constant pressure:

$\mathrm{H}=96.34 \mathrm{Btu} / 1 \mathrm{~b}+.2854 \mathrm{Btu} / \mathrm{lb}=96.63 \mathrm{Btu} / 1 \mathrm{~b}$

$\mathrm{P}=584 \mathrm{psi}$ as before

6.3.3.3 Lift Work From 2,280 Feet

- $\Delta \mathrm{H}=2280 \mathrm{ft} / 778.3 \mathrm{Btu} / \mathrm{ft}-\mathrm{Ib}=2.93 \mathrm{Btulb}$

- Compute friction loss as in incompressible fluid and convert to heat energy:

$$
\Delta \mathrm{H}=\frac{40 \mathrm{psi} \times 144 \mathrm{in}^{2} / \mathrm{ft}^{2}}{778.3 \mathrm{Btu} / \mathrm{ft}^{2}-1 \mathrm{~b} \times 26.1\left(\mathrm{P}_{\mathrm{AV}}\right)}=.284 \mathrm{Btu} / \mathrm{lb}
$$

- Required isentropic arop for ideal lift work plus friction loss:

$$
\Delta \mathrm{H}=2.93 \mathrm{Btu} / \mathrm{lb}+.284 \mathrm{Btu} / \mathrm{lb}=3.21 \mathrm{Btu} / \mathrm{lb}
$$

- Diagram above:

$\mathrm{H}=96.63 \mathrm{Btu} / \mathrm{Ib}-3.2 \mathrm{Btu} / \mathrm{Ib}=93.42 \mathrm{Btu} / \mathrm{Ib}$

$\mathrm{p}=256$ (by diagram)

- Diagram irreversibility:

$$
\begin{aligned}
& \mathrm{H}=93.42 \mathrm{Btu} / \mathrm{lb}+.284 \mathrm{Btu} / 1 \mathrm{~b}=93.70 \mathrm{Btu} \mathrm{lb} \\
& \mathrm{P}=256 \mathrm{psi} \text { as before }
\end{aligned}
$$


- Remaining $\Delta \mathrm{H}^{+}$expanding from 256 to 42 psi:

${\Delta \mathrm{H}_{\text {TURB }}^{\prime}}_{\text {TUR }}=93.70 \mathrm{Btu} / 1 \mathrm{~b}-83.83 \mathrm{Btu} / 1 \mathrm{~b}=9.87 \mathrm{Btu} / 1 \mathrm{~b}$

6.3.3.4 Ideal Energy Split Between Pelton Wheel and Main Turbine

- Combined energy from two-phase flow :

$\Delta \mathrm{H}^{\circ}=9.87 \mathrm{Btu} / \mathrm{lb}$

$E=9.87 \mathrm{Btu} / \mathrm{lb} \times 640.4 \mathrm{lb} / \mathrm{sec}=6321 \mathrm{Btu} / \mathrm{sec}$

- Iiquid flow:

Flow ratio from diagram $=.1719 / 1$

$\dot{w}($ IIQ $)=640.4 \mathrm{lb} / \mathrm{sec} \times .1719 \mathrm{lb} / \mathrm{sec}=110.1 \mathrm{lb} / \mathrm{sec}$

$\Delta \mathrm{H}^{\prime}$ (from diagram) $=65.19 \mathrm{Btu} / 1 \mathrm{~b}-61.02 \mathrm{Btu} / 1 \mathrm{~b}=4.17 \mathrm{Btu} / 1 \mathrm{~b}$

- Ideal Pelton wheel energy rate

$\mathrm{E}=110.1 \mathrm{lb} / \mathrm{sec} \times 4.17 \mathrm{Btu} / \mathrm{lb}=459 \mathrm{Btu} / \mathrm{sec}$

- Ideal main turbine energy

$\mathrm{E}=6321 \mathrm{lb} / \mathrm{sec}-459 \mathrm{Btu} / \mathrm{sec}=5862 \mathrm{Btu} / \mathrm{sec}$

\subsubsection{Generator Output}

- Pelton wheel

$\frac{459 \mathrm{Btu} / \mathrm{sec} \times .7 \text { (Eff. Wheel) } \times .96 \text { (Eff. Gen.) }}{.9482 \mathrm{~kW} \mathrm{sec} / \mathrm{Btu}}=325.3 \mathrm{~kW}$

- Main turbine

$\frac{5862 \mathrm{Btu} / \mathrm{sec} \times .85 \text { (Turb. Eff.) } \times .96 \text { (Gen. Eff.) }}{.9482 \mathrm{Btu} / \mathrm{kW} \mathrm{sec}}=5045 \mathrm{~kW}$

- Total generator output

$5045 \mathrm{~kW}+325 \mathrm{~kW}=5370 \mathrm{~kW}$ 


\subsubsection{Condenser Parasitic Load}

- Turbine exit enthalpy:

$\mathrm{H}=83.84 \mathrm{Btu} / 1 \mathrm{~b}+9.94 \mathrm{Btu} / 1 \mathrm{~b} \times .15$ (Eff. Loss) $=85.33 \mathrm{Btu} / \mathrm{lb}$

- Heat Load

$(85.33-31.3) \mathrm{Btu} / 1 \mathrm{~b} \times 640.4 \mathrm{lb} / \mathrm{sec} \times 3600 \mathrm{sec} \mathrm{hr}$

$=122.2 \times 10^{6} \mathrm{Btu} / \mathrm{hr}$

- Condenser electric load

$$
\frac{122.2 \times 10^{6} \mathrm{Btu} / \mathrm{hr} \times .746 \mathrm{~kW} / \mathrm{hp} \times 21 \mathrm{hp}}{6 \times 10^{6} \mathrm{Btu} / \mathrm{hr} \times .85 \text { (Motor Eff.) }}=375 \mathrm{~kW}
$$

\subsubsection{Busbar Power}

Power (busbar) $=5370 \mathrm{~kW}-375 \mathrm{~kW}=4995 \mathrm{~kW}$

\subsubsection{Conventional Binary Plant Using Isobutane (900 psi)}

6.3.4.1 Organic Parameters

- Flow: $\dot{w}_{0}=160.5\left(w_{B}\right) \times 1.0961=175.91 \mathrm{~b} / \mathrm{sec}$

- Peak conditions: $\mathrm{H}_{0}=594.8 \mathrm{Btu} / \mathrm{lb}$

- $P_{0}=900 \mathrm{psi}, \quad \mathrm{T}_{0}=340.0^{\circ} \mathrm{F}$

- Isentropic drop

$\Delta \mathrm{H}^{\prime}=594.8 \mathrm{Btu} / 1 \mathrm{~b}+635.7 \mathrm{Btu} / \mathrm{lb}=40.9 \mathrm{Btu} / \mathrm{lb}$

\subsubsection{Generator Output}

- Generator output

$40.9 \mathrm{Btu} / \mathrm{lb} \times 175.9 \mathrm{lb} / \mathrm{sec} \times .85$ (Eff. Turb.) $\times .96 \mathrm{Eff.} \mathrm{Gen.)}$ $.9482 \mathrm{Btu} / \mathrm{kW} \cdot \mathrm{sec}$

$=6191 \mathrm{~kW}$ 


\subsubsection{Feed Pump Work}

- Organic volume flow:

$$
Q=\frac{175.9 \mathrm{lb} / \mathrm{sec} \times 60 \mathrm{sec} / \mathrm{min} \times 7.48 \mathrm{gal} / \mathrm{ft}^{3}}{34.49 \mathrm{lb} / \mathrm{ft}^{3}}=2289 \mathrm{gpm}
$$

- Electrical power into feed pump:

$\frac{2289 \mathrm{gpm} \times(920-60) \text { psi } \times 1.015 \text { (Compressibility) }}{2298 \mathrm{gpm} \times \mathrm{psi} / \mathrm{kW} \times .77 \text { (Eff. Turb.) } \times .93 \text { (Eff. Motor) }}=1214 \mathrm{~kW}$

NOTE: Compressibility is approximately $=3.0 \%$. Half of this should be charged to pumping energy.

\subsubsection{Brine Pump Power}

$$
\frac{1300 \mathrm{gpm} \times 755 \mathrm{psi}}{2298 \mathrm{gpm} \cdot \mathrm{psi} / \mathrm{kW} \times 0.51 \text { (Pump Eff.) }}=837 \mathrm{~kW}
$$

\subsubsection{Condenser Parasitic Load}

- $\mathrm{H}_{\mathrm{TURB}}=635.7 \mathrm{Btu}-(40.9 \mathrm{Btu} \times .15 \mathrm{Eff}$. Loss $)=629.6 \mathrm{Btu}$

- $\Delta \mathrm{H}=794 \mathrm{Btu} / \mathrm{lb}-629.6 \mathrm{Btu} / \mathrm{Ib}=164 \mathrm{Btu} / 1 \mathrm{~b}$

- Heat $10 a d=164 \mathrm{Btu} / 1 \mathrm{~b} \times 175.9 \mathrm{lb} / \mathrm{sec} \times 3600 \mathrm{sec} / \mathrm{hr}=99.4 \times 10^{6} \mathrm{Btu} / \mathrm{hr}$

- Condenser load $=\frac{99.4 \times 10^{6} \mathrm{Btu} / \mathrm{hr} \times 21 \mathrm{hp} \times .746 \mathrm{~kW} / \mathrm{hp}}{6 \times 10^{6} \mathrm{Btu} / \mathrm{hr} \times .85 \mathrm{Eff} .}=319 \mathrm{~kW}$

\subsubsection{Busbar Output}

$$
6191 \mathrm{~kW}-1214 \mathrm{~kW}-837 \mathrm{~kW}-319 \mathrm{~kW}=3821 \mathrm{~kW}
$$




\subsubsection{Temperature Rise Due to Feed Pumping}

- Reversible increase in temperature:

$\Delta T=5^{\circ} \mathrm{F}$ (from diagram)

- Temperature increase due to impeller loss:

$P W R=1214 \mathrm{~kW} \times .93 \mathrm{Eff.} \times .23 \mathrm{Eff}$. Loss $=260 \mathrm{~kW}$

$\Delta \mathrm{H}_{\mathrm{lb}}=\frac{260 \mathrm{~kW} \times .9482 \mathrm{Btu} / \mathrm{sec} / \mathrm{kw}}{175.9 \mathrm{lb} / \mathrm{sec}}=1.4 \mathrm{Btu} / \mathrm{lb}$

$\Delta \mathrm{T}=1.4 \mathrm{Btu} / 1 \mathrm{~b} \times .58(\mathrm{Cp})=2.42^{\circ} \mathrm{F}$

- Total temperature increase $=5^{\circ} \mathrm{F}+2.42^{\circ} \mathrm{F}=7.42^{\circ} \mathrm{F}$

- Heat exchanger inlet temperature:

$\mathrm{T}=85^{\circ} \mathrm{F}+7^{\circ} \mathrm{F}=92^{\circ} \mathrm{F}$

\subsubsection{Conventional Binary Plant Using R] ]4 (900 psi)}

\subsubsection{RIl4 Parameters}

$$
\left(\dot{w}_{B}\right) \quad(R)
$$

- Flow: $160.5 \times 3.54868=569.6 \mathrm{lb} / \mathrm{sec}$.

- Peak conditions:

$\mathrm{H}=97.48 \mathrm{Btu} \quad \mathrm{P}_{\mathrm{O}}=900 \mathrm{psi} \quad \mathrm{T}_{\mathrm{O}}=340.0^{\circ} \mathrm{F}$

- Isentropic drop to 42.0 PSIA

- $\Delta \mathrm{H}^{\prime}=97.48 \mathrm{Btu} / \mathrm{Ib}-83.43 \mathrm{Btu} / \mathrm{Lb}=14.05 \mathrm{Btu} / \mathrm{Ib}$

\subsubsection{Generator Output}

- Turbine efficiency considering the $9 \%$ average wetness $=.774$ 
- Generator power:

$14.05 \mathrm{Btu} / \mathrm{lb} \times 569.6 \mathrm{lb} / \mathrm{sec} \times .774 \mathrm{Eff}$. Turb. $\times .96 \mathrm{Eff}$. Gen. $.9482 \mathrm{Btu} / \mathrm{kW} \mathrm{sec}$

$=6271 \mathrm{~kW}$

6.3.5.3 Feed Pump Work

- Organic volume flow at an average density of $89.71 \mathrm{~b} / \mathrm{ft}^{3}$

$Q=\frac{569.6 \mathrm{lb} / \mathrm{sec} \times 60 \mathrm{sec} / \mathrm{min} \times 7.48 \mathrm{gal} / \mathrm{ft}^{3}}{89.68 \mathrm{lb} / \mathrm{ft}^{3}}=2851 \mathrm{gpm}$

- Electric power into feed pump:

$\frac{2851 \mathrm{gpm} \times(920-39) \mathrm{psi} \times 1.012 \text { (Compressibility) }}{2298 \mathrm{gpm} \cdot \mathrm{psi} / \mathrm{kW} \times .77 \text { (Eff. Turb.) } \times .93 \text { (Eff. Motor) }}=1544 \mathrm{~kW}$

NOTE: Compressibility is approximately 2.58 by diagram.

Half of this should be charged to pumping energy.

6.3.5.4 Brine Pump Power

$1300 \mathrm{gpm} \times 755$ PSIA

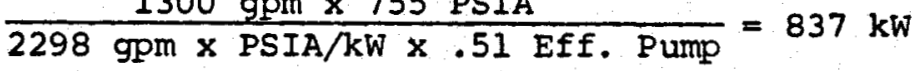

\subsubsection{Condenser Load}

- RI14 enthalpy at turbine exhaust

$\mathrm{H}=83.43 \mathrm{Btu}+(14.05$ Btu $\times .15$ (Eff. Loss) $=85.54 \mathrm{Btu}$

- Heat Load $=\frac{(85.54 \mathrm{Btu}-29.3 \mathrm{Btu})(569.4 \mathrm{lb} / \mathrm{sec} \times 3600 \mathrm{sec} / \mathrm{hr})}{1 \mathrm{~b}}$

$=115.3 \times 10^{6} \mathrm{Btu} / \mathrm{hr}$

- Condenser load $=\frac{115.3 \mathrm{Btu} / \mathrm{hr} \times 10^{6} \times .746 \mathrm{~kW} / \mathrm{hp} \times 21 \mathrm{hp}}{6 \times 10^{6} \mathrm{Btu} / \mathrm{hr} \times .85 \text { (Eff. Motor) }}=354 \mathrm{~kW}$ 
6.3.5.6 Busbar Output

$6271 \mathrm{~kW}-1544 \mathrm{~kW}-837 \mathrm{~kW}-354 \mathrm{~kW}=3536 \mathrm{~kW}$

6.3.5.7 Temperature Rise Due to Feed Pumping

- Reversible increase in temperature

$\Delta T=5^{0} \mathrm{~F}$ (from diagram)

- Temperature increase to impeller loss:

$P W R=1474 \mathrm{~kW} \times .93 \mathrm{Eff}$. Motor $\times .23 \mathrm{Eff}$. Loss $=315 \mathrm{~kW}$

$\Delta \mathrm{H}_{\mathrm{lb}}=\frac{274 \mathrm{~kW} \times .9482 \mathrm{Btu} / \mathrm{kW} \mathrm{sec}}{569.6 \mathrm{ib} / \mathrm{sec}}=.52 \mathrm{Btu} / \mathrm{lb}$

$\Delta T=.46 \mathrm{Btu} / \mathrm{lb} / .22(\mathrm{Cp})=2.36^{\circ} \mathrm{F}$

- Total temperature increase $=5^{\circ} \mathrm{F}+2^{\circ} \mathrm{F}=7^{\circ} \mathrm{F}$

- Temperature into heat exchanger:

$T=85^{\circ} \mathrm{F}+7^{\circ} \mathrm{F}=92^{\circ} \mathrm{F}$

\subsubsection{Brine Pumping Requirements}

\subsubsection{Assumptions and Conditions}

- Ignore 2.000 PPM solids; consider brine to be pure water.

- Temperature at pump setting $=360^{\circ} \mathrm{F}$.

- No loss of heat to formation.

- Pumped flow $=1,300 \mathrm{gpm}$ at density of $55.2 \mathrm{1b} / \mathrm{ft}^{3}$.

- Depth to top of PERFS in production - 5,000 ft.

- Hot column shut-in pressure in production well - 153 PSIA.

- Pump efficiencies:

$-\mathrm{RC}$ shaft $=.70$ (impeller) $\times .92$ (electrical) $\times .79$ (mechanical) $=.51$

- GH turbine pump $=.56 \quad$ (lab tests show .60). 
- Required pressure at well head:

$$
\begin{aligned}
& P(\mathrm{RC})=153\left(\mathrm{P}_{\mathrm{SAT}}\right)+70\left(\mathrm{CO}_{2}\right)=223 \mathrm{PSIA} \\
& \mathrm{P}(\mathrm{GH})=1.0\left(\mathrm{P}_{\mathrm{SAT}}\right)+\approx 5\left(\mathrm{CO}_{2}\right)=6 \mathrm{PSIA}
\end{aligned}
$$

- Production index $-2.4 \mathrm{gpm} / \mathrm{psi}$

- Reinjection index - $5.0 \mathrm{gpm} / \mathrm{psi}$

- Depth to top of PERFS in reinjection well - 5,000 ft.

- Formation temperature top of PERFS $=250^{\circ} \mathrm{F}$

- Static cold column pressure at top of PERFS in reinjection well

$P=5,000$ ft $\times \frac{60.89\left(P_{A V}\right)}{144}=2114 \mathrm{psi}$

( $P$ at average temperature $-165^{\circ} \mathrm{F}$ )

\subsubsection{Conventional Binary Plant Requirements}

- Flow losses

$\begin{array}{lll}\text { Production well } & 44 \mathrm{psi} & \text { Calculated } \\ \text { Heat Exchanger } & 20 \mathrm{psi} & \text { Estimated } \\ \text { Reinjection Line } & 30 \mathrm{psi} & \text { Estimated } \\ \text { Reinjection well } & 40 \mathrm{psi} & \text { Calculated }\end{array}$

- Volumetric flow in reinjection well

$$
Q=\frac{160.5(\dot{w}) \times 7.48\left(\mathrm{gal} / \mathrm{ft}^{3}\right) \times 60(\mathrm{sec} / \mathrm{min})}{60.99\left(\mathrm{P} \text { at } 160^{\circ} \mathrm{F}\right)}=1181 \mathrm{gpm}
$$

- Column head during reinjection

$$
\mathrm{P}=\frac{5000 \mathrm{ft} \times 60.99(\mathrm{P})}{144}=2118 \mathrm{psi}
$$


- Pump pressure addition required

$\Delta \mathrm{P}=\frac{1300 \mathrm{gpm}}{2.4 \text { (P.I.) }}-153$ (well head pressure)

+44 (well loss) + 20 ( $\mathrm{H}_{x}$ loss) +2114 (static column)

- 2118 (reinjection column) +30 (line) + 40 (we1l loss)

$+\frac{1181 \mathrm{gpm}}{5.0 \text { (P.I.) }}$

$=755 \mathrm{psi}$

- Check production well head margin

$P($ well head $)=153$ (static hot column) +755 (pump $\Delta P$ )

$-\frac{1300 \mathrm{gpm}}{2.4 \text { (P.I.) }}-44$ (well loss)

$=322 \mathrm{psi}$

Margin $=322-223$ (required) $=99 \mathrm{psi}$

6.3.6.3 Gravity Head Plant

- Flow and head losses

Production Well $(5,000$ to 2,300$)$

24 psi

Heat Exchanger

50 psi

Head Loss in $\mathrm{H}_{x}$ (due to cooling of brine)

37 psi

Reinjection Line

30 psi

Reinjection Well

40 psi

- Volumetric flow in reinjection well

$Q=\frac{160.5(\mathrm{w}) \times 7.48\left(\mathrm{gal} / \mathrm{ft}^{2}\right) \times 60(\mathrm{sec} / \mathrm{min})}{61.83\left(\mathrm{P}-112^{\circ} \mathrm{F}\right)}=1165 \mathrm{gpm}$ 
- Column head during reinjection

$P=\frac{5000(\mathrm{ft}) \times 61.83(\mathrm{P})}{144}=2147 \mathrm{psi}$

- Pump pressure addition required

$\Delta \mathrm{P}=\frac{1300 \mathrm{gpm}}{2.4(\mathrm{P} . \mathrm{I} .)}-153$ (well head) +24 (well loss)

$+50\left(\mathrm{H}_{\mathrm{x}}\right.$ loss $)+37$ (head loss) +30 (line)

- 2147 (reinjection column) + 2114 (static column)

+39 (well loss) $+\frac{1165 \mathrm{gpm}}{5.0 \text { (P.I.) }}$

$=767 \mathrm{psi}$

- Check margin

$\mathrm{P}($ well head $)=153$ (static column) +767 (Pump) $-\frac{1300 \mathrm{gpm}}{2.4 \text { (P.I.) }}$

$-24($ well $)-50\left(\mathrm{H}_{\mathrm{x}}\right)-37$ (head)

$=114 \mathrm{psi}-$ required $6.0 \mathrm{psi}$

6.3.6.4 Necessary pump setting depths

- Flow loss from $5,000 \mathrm{ft}$ to $2,280 \mathrm{ft}=28 \mathrm{psi}$

- Required inlet pressure above saturation to provide for suction specific speed of 4,500

R.C. $=15 \mathrm{psi}$

G.H. $=140$ psi

- Inlet pressures

$$
\begin{aligned}
& \mathrm{P}(\mathrm{RC})=223+15=238 \mathrm{psi} \\
& \mathrm{P}(\mathrm{GH})=223+140=363 \mathrm{psi}
\end{aligned}
$$


- RC depth required

$2(R C)=[283$ (inlet) +27 (well p to $1900 \mathrm{ft})]+\frac{1300 \mathrm{gpm}}{2.4 \text { (P.I.) }}$

-153 (well head) $\left(\frac{144}{55.22(P)}\right)$

$=1704$

- $\mathrm{GH}$ depth required

$2(\mathrm{GH})+\left(363+28+\frac{1300}{2.4}-153\right)\left(\frac{144}{55.22(\mathrm{P})}\right)$

$=2033$

- RC setting depth

Add 70 psi margin to provide for observed variability in $\mathrm{CO}_{2}$ content $z=(1740+70) \times \frac{144}{55.22}=1887 \mathrm{ft}$

GH pressure margin

$\Delta \mathrm{P}=(2280-2033) \times \frac{55.22}{144}=95 \mathrm{psi}$ 


\subsection{CONCLUSIONS}

The Gravity Head System of well-head generating units shows clearly superior rates of return on investment over conventional binary systems. The economic advantages of the Gravity Head system stem from two major characteristics: 1) lower cost per installed $\mathrm{kW}$ of net generating capacity, and 2) higher net $\mathrm{kW}$ output per pound of geothermal brine utilized. It can be argued that if the brine charges were based solely on Btus extracted rather than on pounds of brine utilized, a part of the Gravity Head system economic advantage would disappear. However, it does not seem reasonable that for a pressure-dominated reservoir such as East Mesa, where the useful life of the resource is limited by the volume of brine (rather than head) extracted, the brine charge for a given temperature resource should be based on anything other than volume utilized.

In any event, for reservoirs which are temperature dominated and a brine charge based on Btus rather than volume extracted is justified, or where there are minimum reinjection temperature constraints, a less efficient and less expensive version of the Gravity Head system would be indicated. In such a case, the loss in economic advantage for superior volumetric efficiency would be at least partially recaptured by lowered capital cost.

Finally, it must be recognized that the economic advantages indicated in this report can be realized only if the downhole equipment is made to operate reliably. For this reason, a great deal of research into bearings, seals, lubricants, etc. has been undertaken under this contract in order to achieve a composite mean-time-between-failure in excess of twelve months. This research, combined with the field experience acquired in inserting and withdrawing dumy heat exchanges from well 87-6, plus the successful operation of the automated welding and automated arc-air weld removal systems which were developed under this contract, supports the conclusion that reliable operation and efficient installation and removal of the downhole equipment are imminently achievable. 


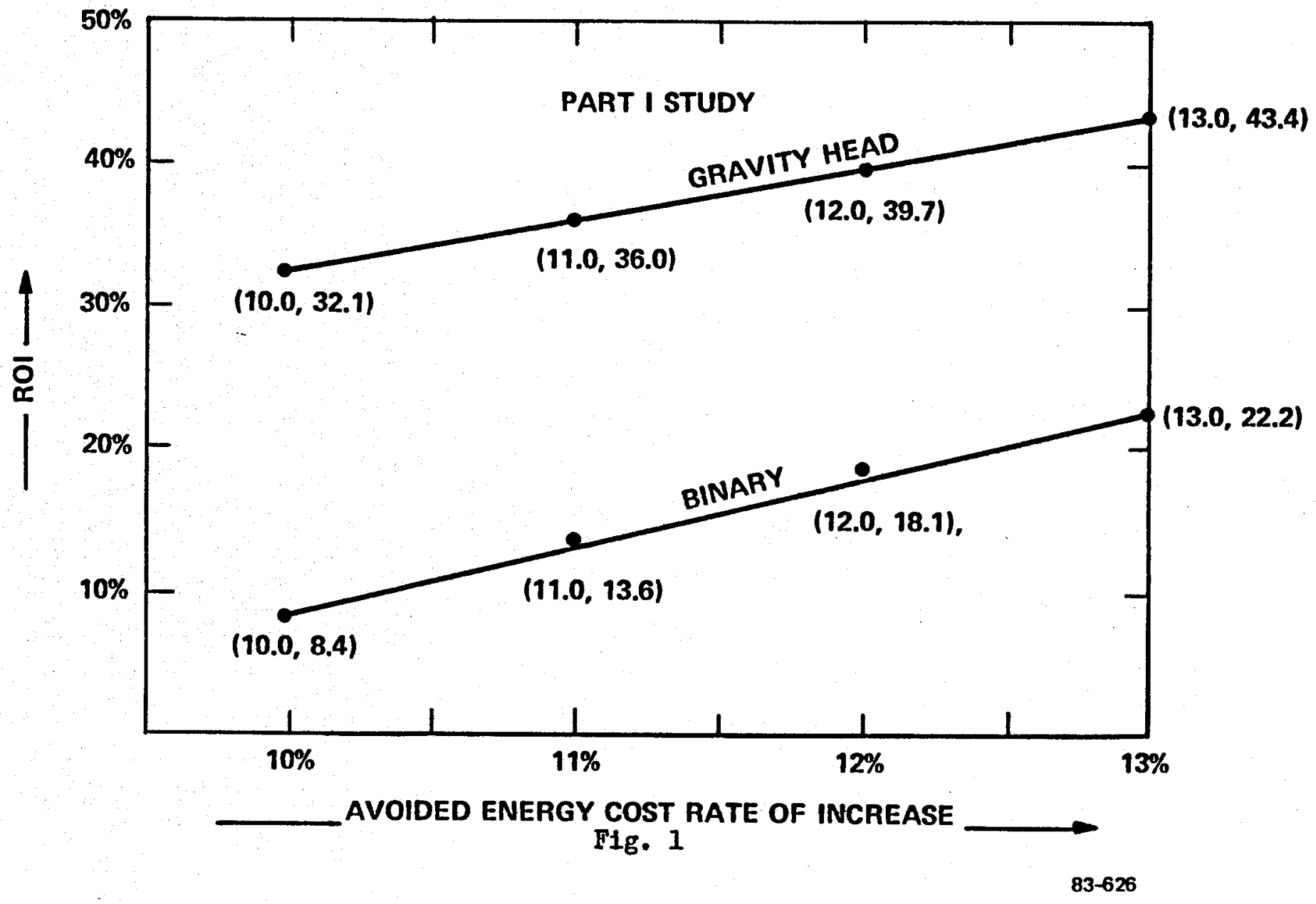

Vol. VI, Fig. I 


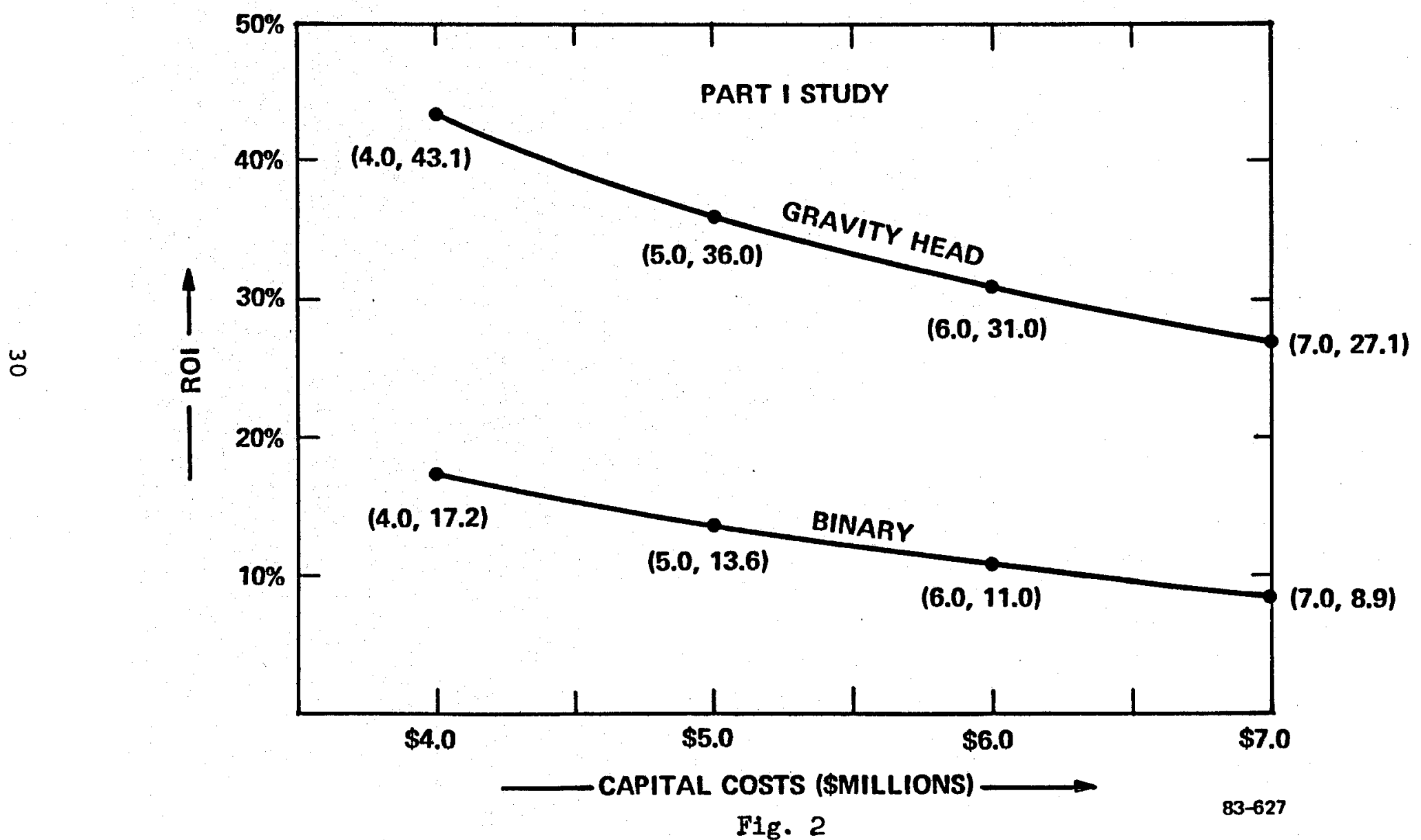

Vol. VI, Fig. 2 


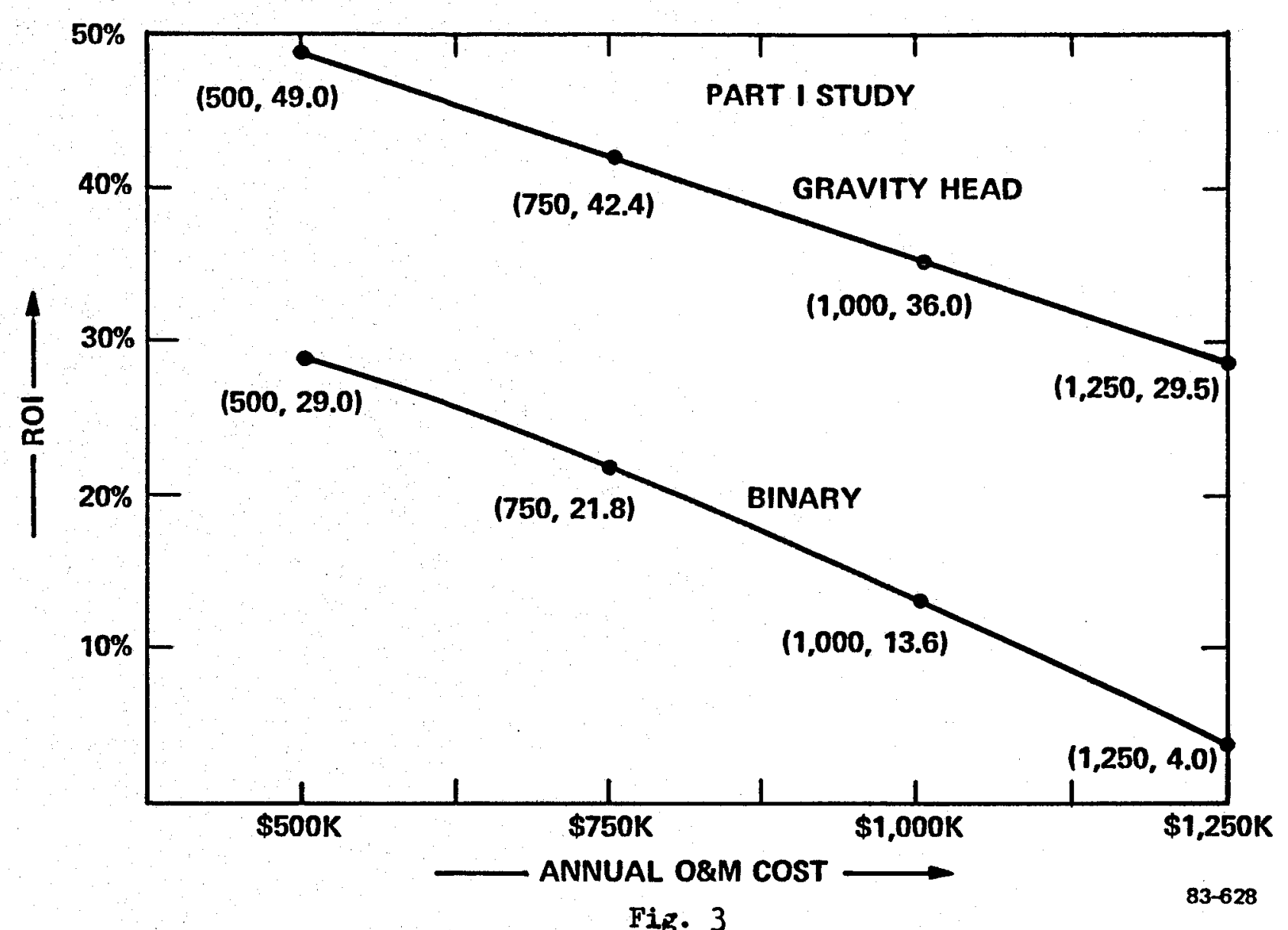

Vol. VI, Fig. 3 


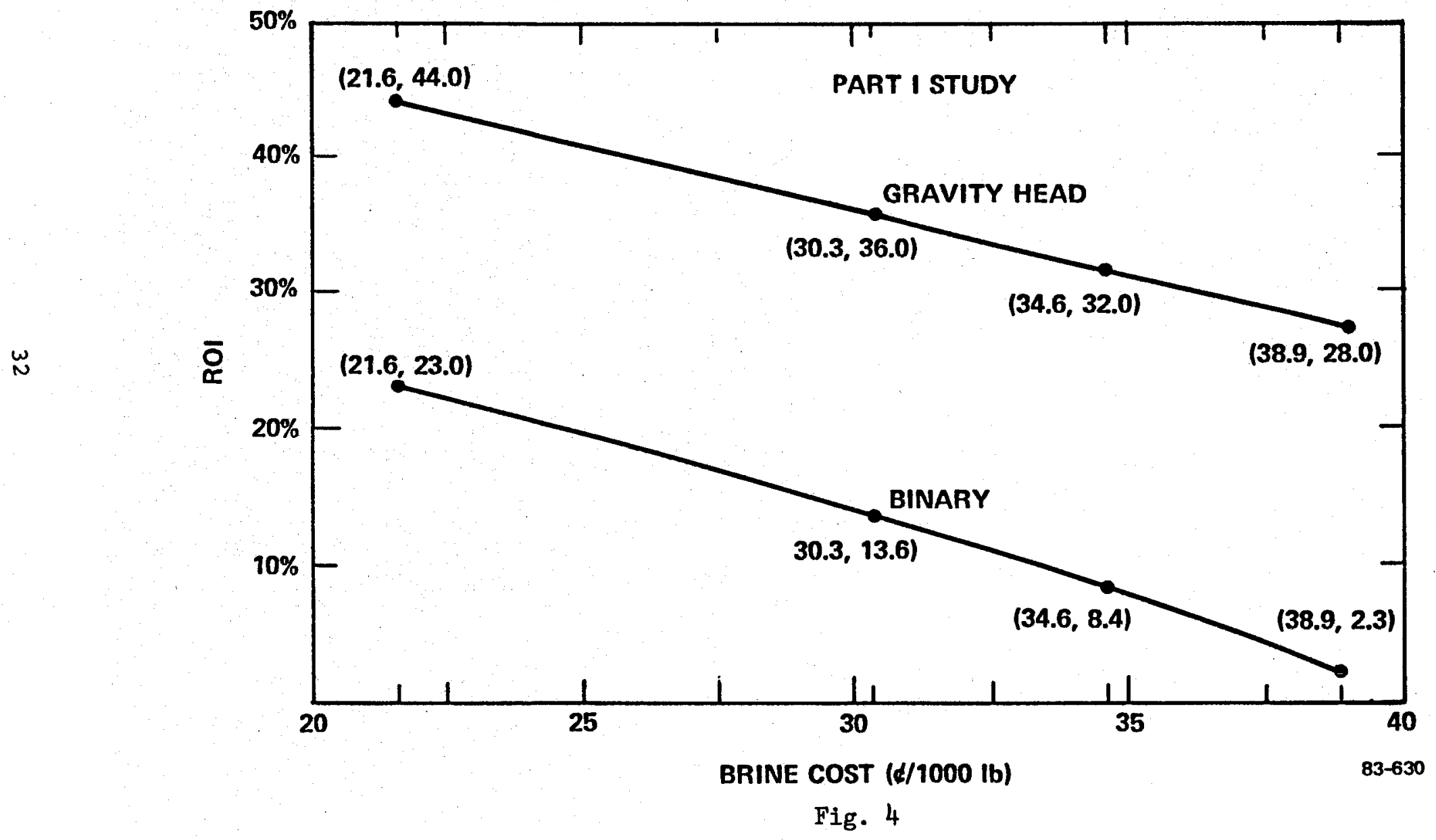

Vol. VI, Fig. 4 
PART II STUDY

(FIVE-UNIT PLANTS WITH WELLS INCLUDED)

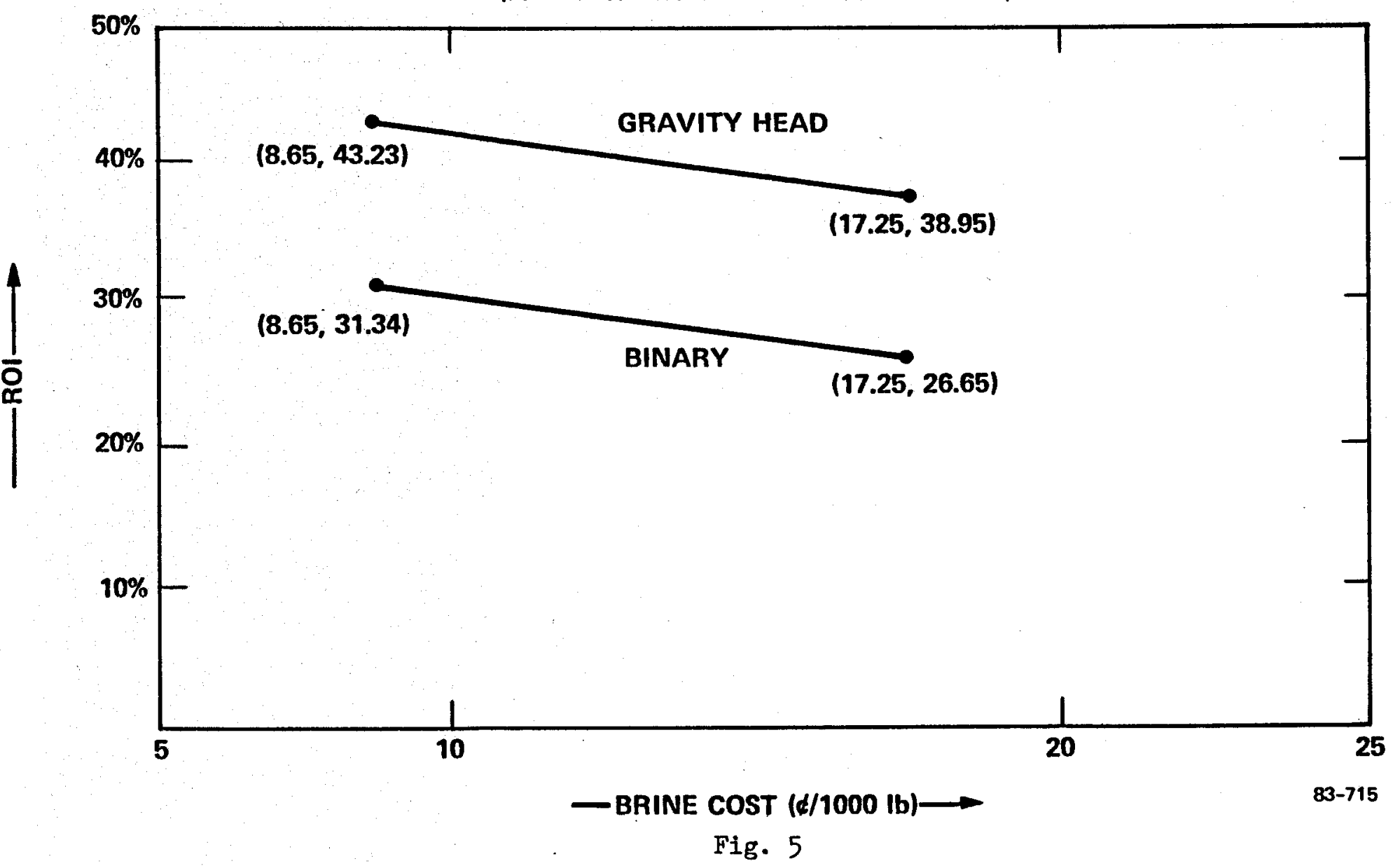

Vol. VI, Fig. 5 


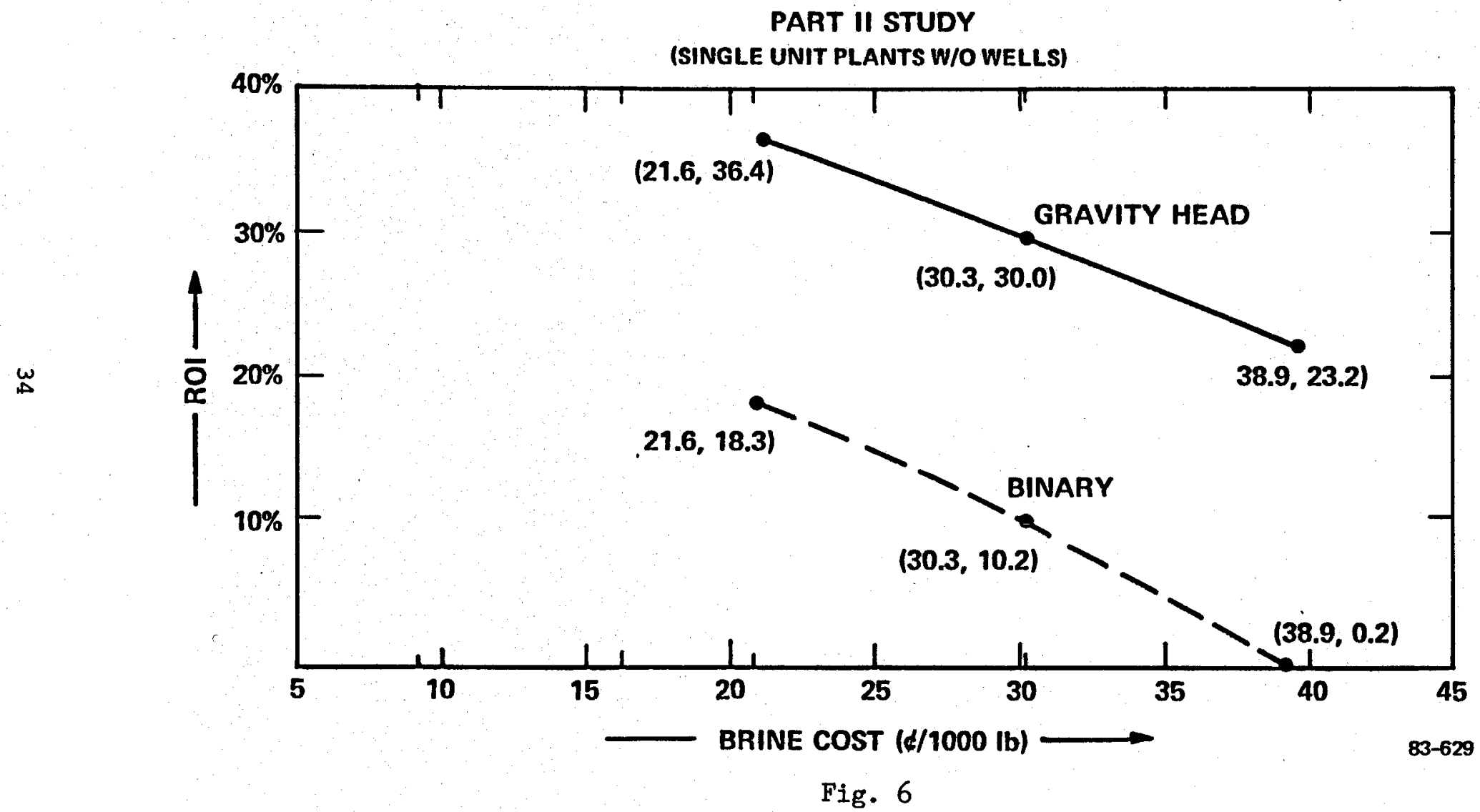

Vol VI, Fig. 6 
APPENDIX A

CASH FLOW ANALYSIS

CHARTS 


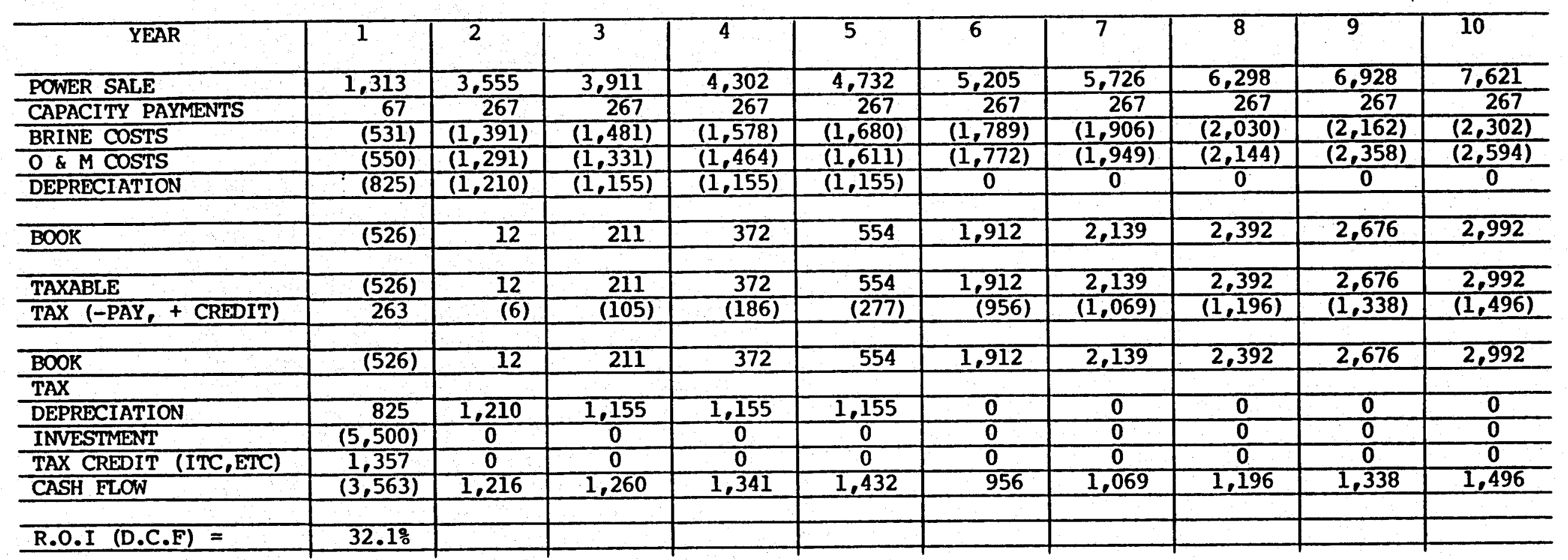

PART I STUDY --10\% elec. escalator--Gravity Head 


\begin{tabular}{|c|c|c|c|c|c|c|c|c|c|c|}
\hline YEAR & 1 & 2 & 3 & 4 & 5 & 6 & 7 & 8 & 9 & 10 \\
\hline POWER SALE & 1,361 & 3,753 & 4,203 & 4,707 & 5,272 & 5,905 & 6,614 & 7,407 & 8,296 & 9,292 \\
\hline CAPACITY PAYMENTS & 67 & 267 & 267 & 267 & 267 & 267 & 267 & 267 & 267 & 267 \\
\hline BRINE COSTS & (531) & $(1,391)$ & $(1,481)$ & $(1,578)$ & $(1,680)$ & $(1,789)$ & $(1,906)$ & $(2,030)$ & $(2,162)$ & $(2,302)$ \\
\hline$O \& M$ COSTS & $(550)$ & $(1,210)$ & $(1,331)$ & $(1,464)$ & $(1,611)$ & $(1,772)$ & $(1,949)$ & $(2,144)$ & $(2,358)$ & $(2,594)$ \\
\hline DEPRECIATION & $(825)$ & $(1,210)$ & $(1,155)$ & $(1,155)$ & $(1.155)$ & 0 & 0 & 0 & 0 & 0 \\
\hline $\mathrm{BOOK}$ & $(478)$ & 209 & 503 & 778 & 1,094 & 2,611 & 3,026 & 3,501 & 4,044 & 4.663 \\
\hline TAXABLE & & & & & & & & & & \\
\hline $\begin{array}{l}\text { TAXABLE } \\
\text { TAX (-PAY, + CREDIT })\end{array}$ & $\frac{(478)}{239}$ & 209 & 503 & 778 & 1,094 & 2,611 & 3,026 & 3,501 & 4.044 & $\frac{4,663}{12,2221}$ \\
\hline & 239 & (105) & (251) & (389) & (547) & $(1,306)$ & $(1,513)$ & $(1,751)$ & $(2,022)$ & $(2,332)$ \\
\hline$\frac{\mathrm{BOOK}}{\mathrm{TAX}}$ & $(478)$ & 209 & 503 & 778 & 1,094 & 2,611 & 3,026 & 3,501 & 4.044 & 4.663 \\
\hline INVESTMENT & $\frac{825}{(5,500)}$ & 1,210 & 1,155 & 1,155 & 1,155 & 0 & 0 & 0 & 0 & 0 \\
\hline TAX CREDIT (ITC,ETC) & $(5,500)$ & 0 & 0 & 0 & 0 & 0 & 0 & 0 & 0 & 0 \\
\hline CASH ELOW & $\frac{1,375}{(3,539)}$ & 0 & 0 & 0 & 0 & 0 & 0 & 0 & 0 & 0 \\
\hline & $(3,539)$ & 1,315 & 1,406 & 1,544 & 1,702 & 1,306 & 1,513 & 1,751 & 2,022 & 2,332 \\
\hline R.O.I (D.C.F) $=$ & $39.7 \%$ & & & & & & & & & \\
\hline & & & & & & & & & & \\
\hline
\end{tabular}

PART I STUDY - $-12 \%$ elec. escalator --Gravity Head 


\begin{tabular}{|c|c|c|c|c|c|c|c|c|c|c|}
\hline YEAR & 1 & 2 & 3 & 4 & 5 & 6 & 7 & 8 & 9 & 10 \\
\hline POWER SALE & 1,386 & 3,854 & 4,355 & 4,921 & 5,561 & 6,284 & 7,101 & 8,024 & 9,067 & 10,246 \\
\hline CAPACITY PAYMENTS & 67 & 267 & 267 & 267 & 267 & 267 & 267 & .267 & 267 & 267 \\
\hline BRINE COSTS & (531) & $(1,391)$ & $(1,481)$ & $(1,578)$ & $(1,680)$ & $(1,789)$ & $(1,906)$ & $(2,030)$ & $(2,162)$ & $(2,302)$ \\
\hline $0 \& M$ COSTS & $(550)$ & $(1,210)$ & $(1,331)$ & $(1,464)$ & $(1,611)$ & $(1,772)$ & $(1,949)$ & $(2,144)$ & $(2,358)$ & $(2,594)$ \\
\hline DEPRECIATION & $(825)$ & $(1,210)$ & $(1,155)$ & $(1,155)$ & $(1,155)$ & 0 & 0 & 0 & 0 & 0 \\
\hline BOOK & (453) & 310 & 655 & 992 & 1,383 & 2,990 & 3,514 & 4,118 & 4,815 & 5,617 \\
\hline TAXABLE & $(453)$ & 310 & 655 & 992 & 1,383 & 2,990 & 3,514 & 4,118 & 4,815 & 5,617 \\
\hline TAX (-PAY, + CREDIT) & 227 & (155) & $(328)$ & $(496)$ & (691) & $(1,495)$ & $(1,757)$ & $(2,059)$ & $(2,408)$ & $(2,809)$ \\
\hline $\mathrm{BOOK}$ & (453) & 310 & 655 & 992 & 1,383 & 2,990 & 3,514 & 4,118 & 4,815 & 5,617 \\
\hline TAX & & & & & & & & & & \\
\hline DEPRECIATION & 825 & 1,210 & 1,155 & 1,155 & 1,155 & $\mathbf{0}$ & $\overline{0}$ & $\mathbf{0}$ & $\overline{0}$ & $\mathbf{0}$ \\
\hline INVESTMENT & $(5,500)$ & 0 & 0 & 0 & 0 & 0 & $\overline{0}$ & $\overline{0}$ & 0 & 0 \\
\hline TAX CREDIT (ITC,ETC) & 1,375 & 0 & 0 & 0 & 0 & 0 & 0 & 0 & 0 & 0 \\
\hline CASH FLOW & $(3,527)$ & 1,365 & 1,483 & 1,651 & 1,846 & 1,495 & 1,757 & 2,059 & 2,408 & 2,809 \\
\hline R.O.I (D.C.F) $=$ & $43.4 \%$ & & & & & & & & & \\
\hline
\end{tabular}

PART I STUDY - $13 \%$ elec. escalator-Gravity Head 


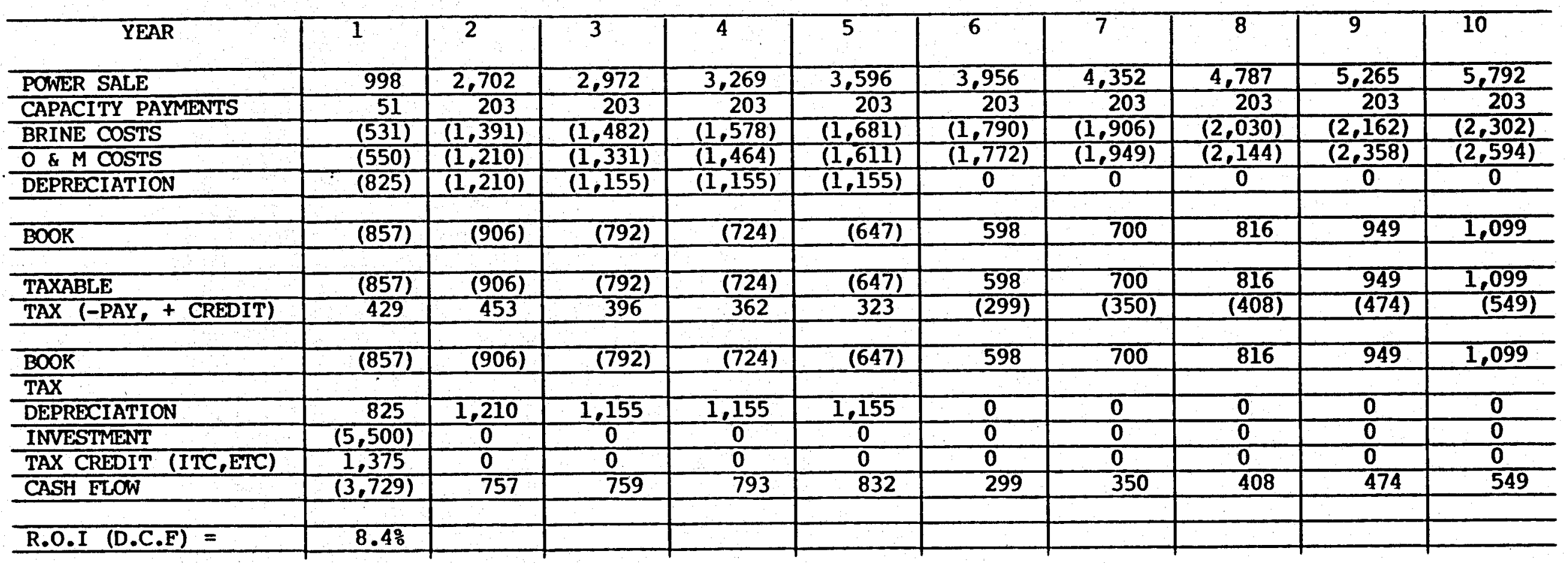

PART I STUDY - 10\% ELEC. ESCALATOR - BINARY 


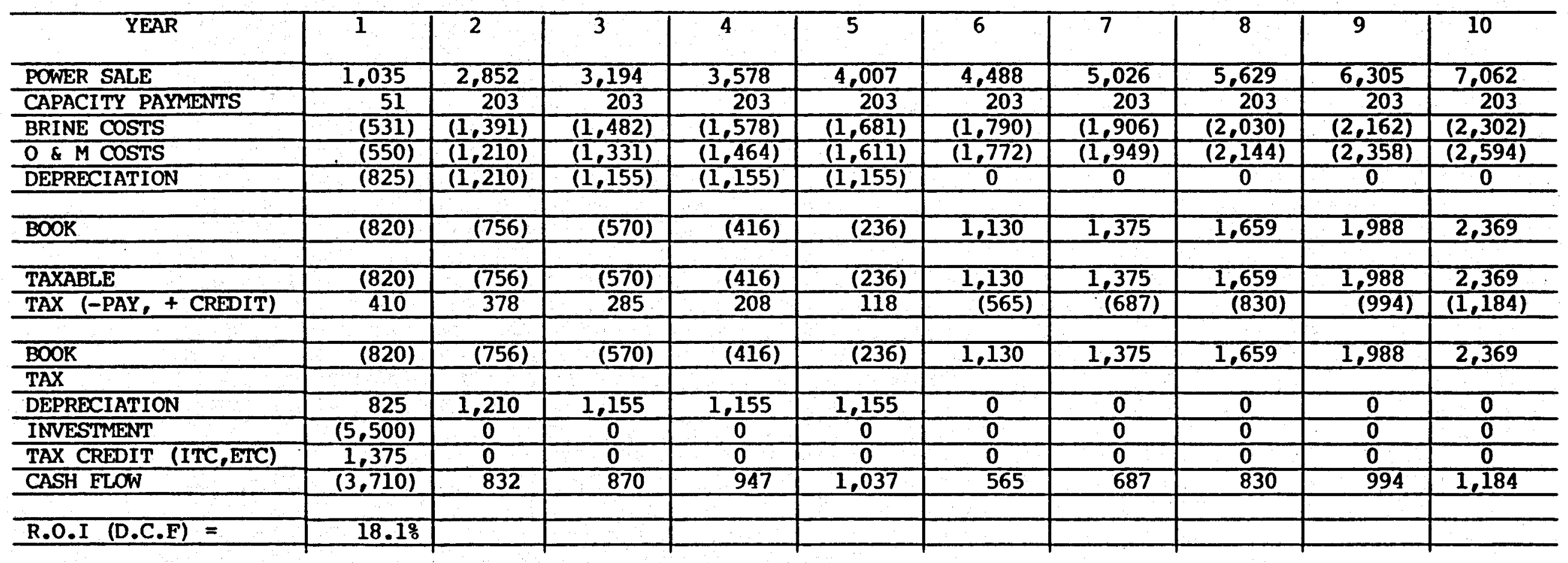

PART I STUDY - 12\% ELEC. ESCALATOR - BINARY 


\begin{tabular}{|c|c|c|c|c|c|c|c|c|c|c|}
\hline YEAR & 1 & 2 & 3 & 4 & 5 & 6 & 7 & 8 & 9 & 10 \\
\hline POWER SALE & 1,053 & 2,929 & 3,310 & 3,740 & 4.226 & 4,776 & 5,397 & 6,098 & 6,891 & 7,787 \\
\hline CAPACITY PAYMENTS & 51 & 203 & 203 & 203 & 203 & 203 & 203 & 203 & 203 & 203 \\
\hline BRINE COSTS & $(531)$ & $(1,391)$ & $(1,482)$ & $(1,578)$ & $(1,681)$ & $(1,790)$ & $(1,906)$ & $(2,030)$ & $(2,162)$ & $(2,302)$ \\
\hline O\& M COSTS & $(550)$ & $(1,210)$ & $(1,331)$ & $(1,464)$ & $(1,611)$ & $(1,772)$ & $(1,949)$ & $(2,144)$ & $(2,358)$ & $(2,594)$ \\
\hline DEPRECIATION & $(825)$ & $(1,210)$ & $(1,155)$ & $(1,155)$ & $(1,155)$ & 0 & 0 & 0 & 0 & 0 \\
\hline $\mathrm{BOOK}$ & $(802)$ & $(679)$ & $(455)$ & $(254)$ & $(16)$ & 1,418 & 1,745 & 2,128 & 2,574 & 3,094 \\
\hline & & & & & & & & & & \\
\hline TAXABLE & $(802)$ & $(679)$ & $(455)$ & $(254)$ & (16) & 1,418 & 1,745 & 2,128 & 2,574 & 3,094 \\
\hline TAX $(-\mathrm{PAY},+$ CREDIT $)$ & 401 & 339 & 227 & 127 & 8 & $(709)$ & $(873)$ & $(1,064)$ & $(1,287)$ & $(1,547)$ \\
\hline BOOK & $(802)$ & (679) & $(445)$ & (254) & (16) & 1,418 & 1,745 & 2,128 & 2,574 & 3,094 \\
\hline TAX & & & & & & & & & & \\
\hline DEPRECIATION & 825 & 1,210 & 1,155 & 1,155 & 1,155 & $\mathbf{0}$ & $\mathbf{0}$ & 0 & 0 & 0 \\
\hline INVESTMENT & $(5,500)$ & 0 & 0 & 0 & 0 & $\mathbf{0}$ & 0 & 0 & 0 & 0 \\
\hline TAX CREDIT (ITC,ETC) & 1,375 & 0 & 0 & 0 & 0 & 0 & 0 & 0 & 0 & 0 \\
\hline CASH FLOW & $(3,701)$ & 871 & 928 & 1,028 & 1,147 & 709 & 873 & 1,064 & 1,287 & 1,547 \\
\hline R.O.I (D.C.F) $=$ & $22.2 \%$ & & & & & & & & & \\
\hline
\end{tabular}

PART I STUDY - $13 \%$ ELEC. ESCALATOR - BINARY 


\begin{tabular}{|c|c|c|c|c|c|c|c|c|c|c|}
\hline YEAR & 1 & 2 & 3 & 4 & 5 & 6 & 7 & 8 & $\overline{9}$ & 10 \\
\hline POWER SALE & 1,337 & 3,653 & 4,055 & 4,501 & 4,996 & 5,546 & 6,156 & 6,833 & 7,584 & 8,419 \\
\hline CAPACITY PAYMENTS & 67 & 267 & 267 & 267 & 267 & 267 & 267 & 267 & 267 & 267 \\
\hline BRINE COSTS & (531) & $(1,391)$ & $(1,481)$ & $(1,578)$ & $(1,680)$ & $(1,789)$ & $(1,906)$ & $(2,030)$ & $(2,162)$ & $(2,302)$ \\
\hline O\& M COSTS & $(550)$ & $(1,210)$ & $(1,331)$ & $(1,464)$ & $(1,611)$ & $(1,772)$ & $(1,949)$ & $(2,144)$ & $(2,358)$ & $(2,594)$ \\
\hline \multirow[t]{2}{*}{ DEPRECIATION } & $(660)$ & $(968)$ & (924) & (924) & (924) & 0 & 0 & 0 & 0 & 0 \\
\hline & & & & & & & & & & \\
\hline \multirow[t]{2}{*}{$\mathrm{BOOK}$} & (337) & 351 & 586 & 803 & 1,049 & 2,252 & 2,569 & 2,927 & 3,332 & 3,790 \\
\hline & & & & & & & & & & \\
\hline \multirow{3}{*}{$\begin{array}{l}\text { TAXABLE } \\
\text { TAX (-PAY, + CREDIT) }\end{array}$} & $(337)$ & 351 & 586 & 803 & 1,049 & 2,252 & 2,569 & 2,927 & 3,332 & 3,790 \\
\hline & 168 & $(176)$ & (293) & $(401)$ & (524) & $(1,126)$ & $(1,284)$ & $(1,463)$ & $(1,666)$ & $(1,895)$ \\
\hline & & & & & & & & & & \\
\hline \multirow{2}{*}{ BOOK } & $(337)$ & 351 & 586 & 803 & 1,049 & 2,252 & 2,569 & 2,927 & 3,332 & 3,790 \\
\hline & & & & & & & & & & \\
\hline DEPRECIATION & 660 & 968 & 924 & 924 & 924 & 0 & $\overline{0}$ & 0 & 0 & $\overline{0}$ \\
\hline \multirow{3}{*}{$\begin{array}{l}\text { INVESTMENT } \\
\text { TAX CREDIT (ITC,ETC) } \\
\text { CASH ELOW }\end{array}$} & $(4,400)$ & 0 & 0 & 0 & 0 & 0 & 0 & 0 & 0 & 0 \\
\hline & 1,100 & 0 & 0 & 0 & 0 & 0 & 0 & 0 & 0 & 0 \\
\hline & $(2,808)$ & 1,144 & 1.217 & 1,324 & 1,448 & 1,126 & 1,284 & 1,463 & 1,666 & 1,895 \\
\hline R.O.I (D.C.F) $=$ & $43.1 \%$ & & & & & & & & & \\
\hline
\end{tabular}

PART I STUDY - \$4,000K CAPITAL COST - GRAVITY HEAD 


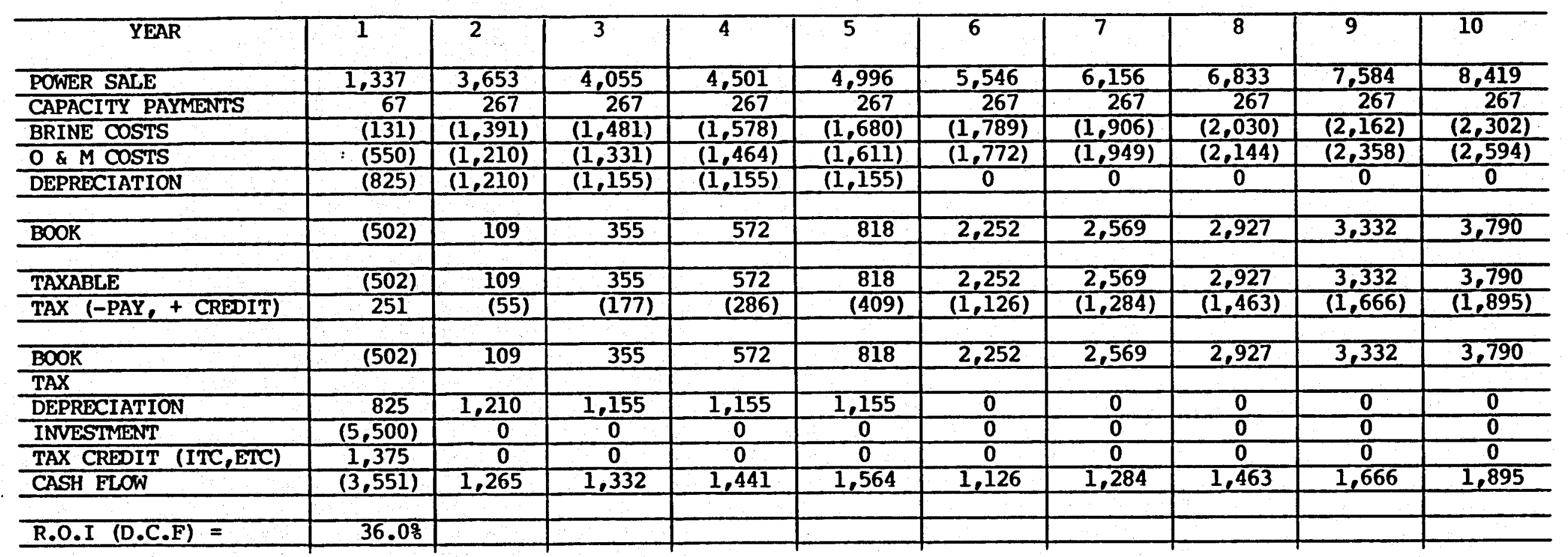




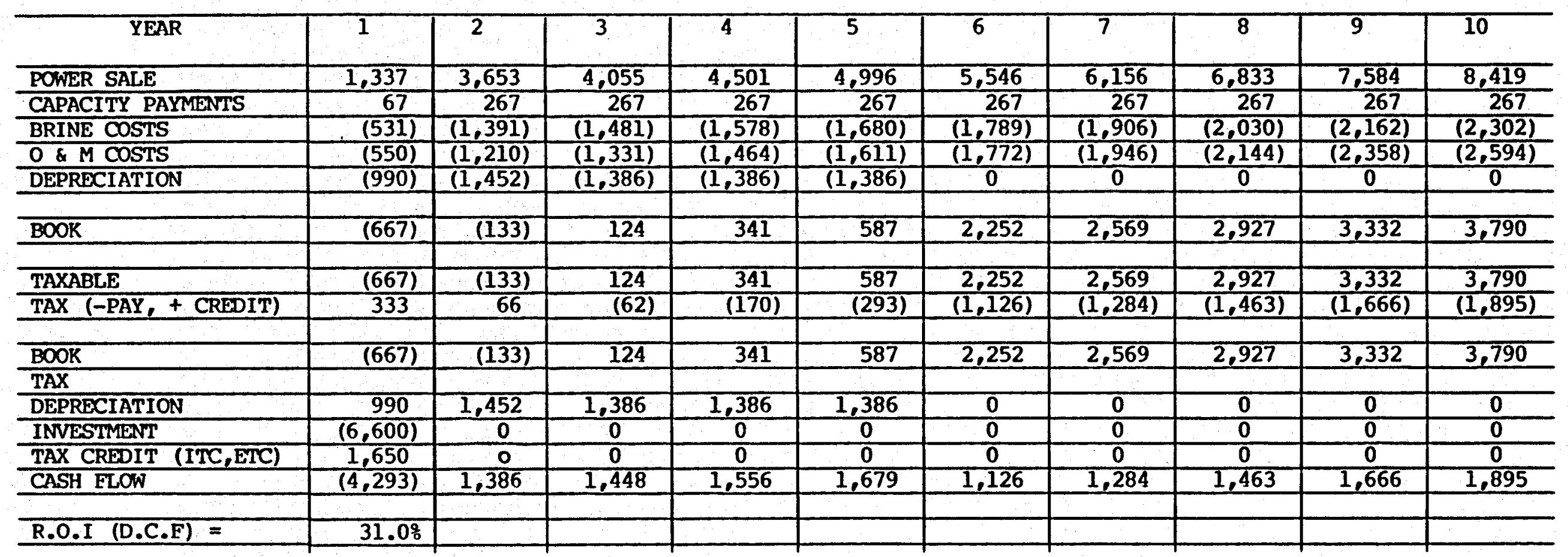

PART I STUDY - $\$ 6,000$ CAPITAL COST GRAVITY HEAD 


\begin{tabular}{|c|c|c|c|c|c|c|c|c|c|c|}
\hline YEAR & 1 & 2 & 3 & 4 & 5 & 6 & 7 & $\overline{8}$ & 9 & 10 \\
\hline POWER SALE & 1,337 & 3,653 & 4,055 & 4,501 & 4,996 & 5,546 & 6,156 & 6,833 & 7.584 & 8,419 \\
\hline CAPACITY PAYMENTS & 67 & 267 & 267 & 267 & 267 & 267 & 267 & 267 & 267 & 267 \\
\hline BRINE COSTS & (531) & $(1,391)$ & $(1,481)$ & $(1,578)$ & $(1,680)$ & $(1,789)$ & $(1,906)$ & $(2,030)$ & $(2,162)$ & $(2,302)$ \\
\hline $0 \& M$ COSTS & $(550)$ & $(1,210)$ & $(1,331)$ & $(1,464)$ & $(1,611)$ & $(1,772)$ & $(1,949)$ & $(2,144)$ & $(2,358)$ & $(2,594)$ \\
\hline DEPRECIATION & $(1,155)$ & $(1,694)$ & $(1,617)$ & $(1,617)$ & $(1,617)$ & 0 & 0 & 0 & 0 & 0 \\
\hline & & & & & & & & & & \\
\hline TAXABLE & $(832)$ & $(375)$ & (107) & 110 & 356 & 2,252 & 2,569 & 2,927 & 3,332 & 3,790 \\
\hline TAX $(-\mathrm{PAY},+$ CREDIT $)$ & 416 & 187 & 54 & (55) & $(178)$ & $(1,126)$ & $(1,284)$ & $(1,463)$ & $(1,666)$ & $(1,895)$ \\
\hline $\mathrm{BOOK}$ & $(832)$ & (375) & (107) & 110 & 356 & 2,252 & 2,569 & 2,927 & 3,332 & 3,790 \\
\hline $\operatorname{TAX}$ & & & & & & & & & & \\
\hline DEPRECIATION & 1,155 & 1,694 & 1,617 & 1,617 & 1,617 & 0 & $\overline{0}$ & 0 & $\mathbf{0}$ & 0 \\
\hline INVESTMENT & $(7,700)$ & 0 & 0 & 0 & 0 & 0 & 0 & $\overline{0}$ & $\mathbf{0}$ & 0 \\
\hline TAX CREDIT (ITC,ETC) & 1,925 & 0 & 0 & 0 & 0 & 0 & 0 & 0 & 0 & 0 \\
\hline CASH FLOW & $(5,036)$ & 1,507 & 1,563 & 1,672 & 1,795 & 1,126 & 1,284 & 1,463 & 1,666 & 1,895 \\
\hline R.O.I (D.C.F) $=$ & $27.1 \%$ & & & & & & & & & \\
\hline
\end{tabular}

PART I STUDY - \$7,000K CAPITAL COST - GRAVITY HEAD 


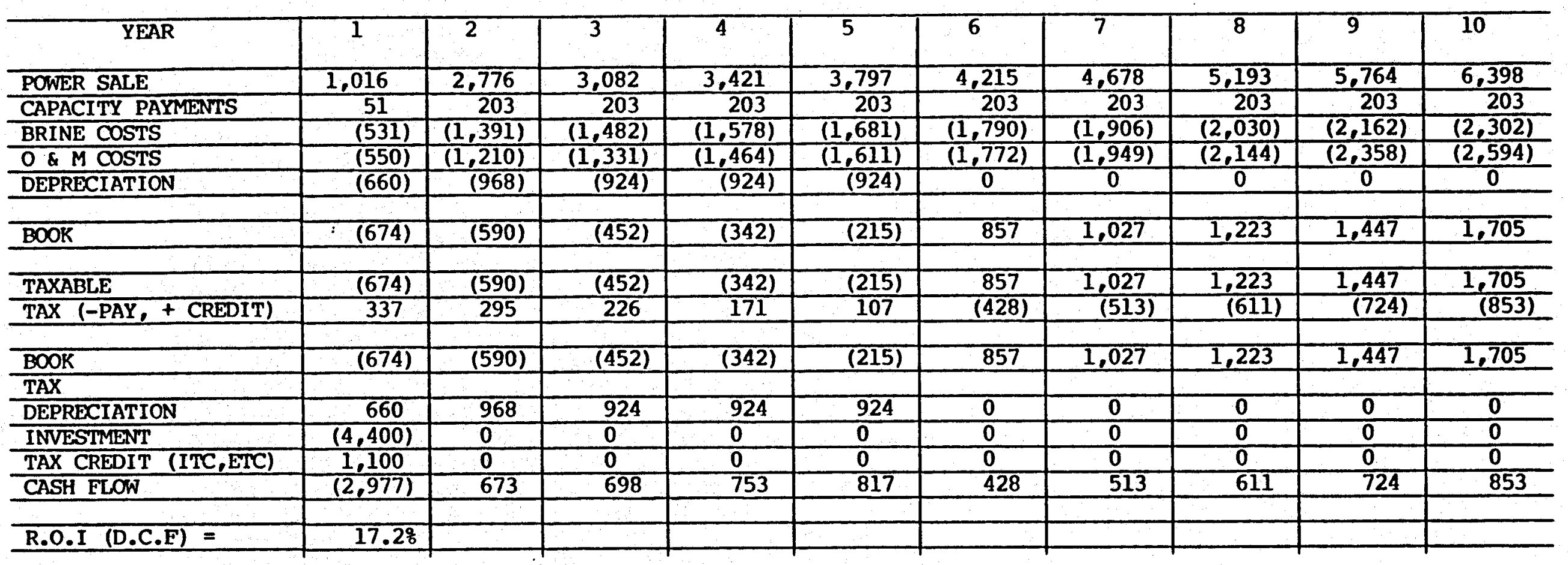

PART I STUDY - \$4,000 K CAPITAL COST - BINARY 


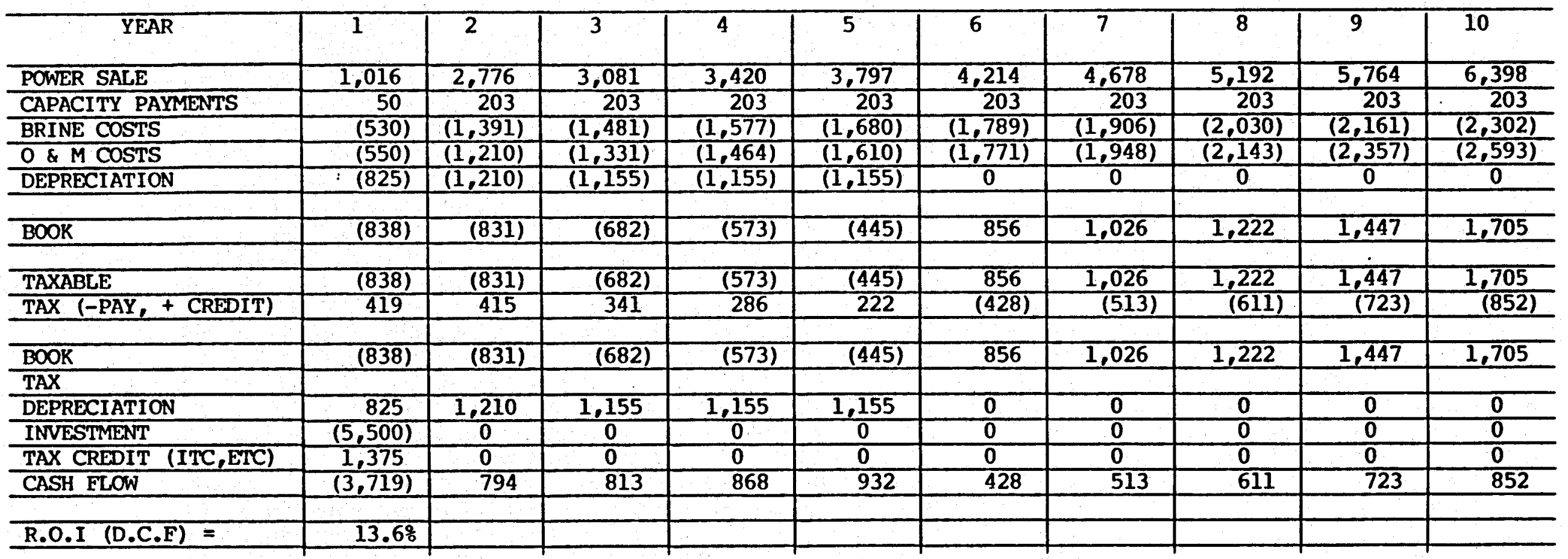

BINARY

PART I STUDY - \$5,000K CAPITAL COST BASE LINE CASE 


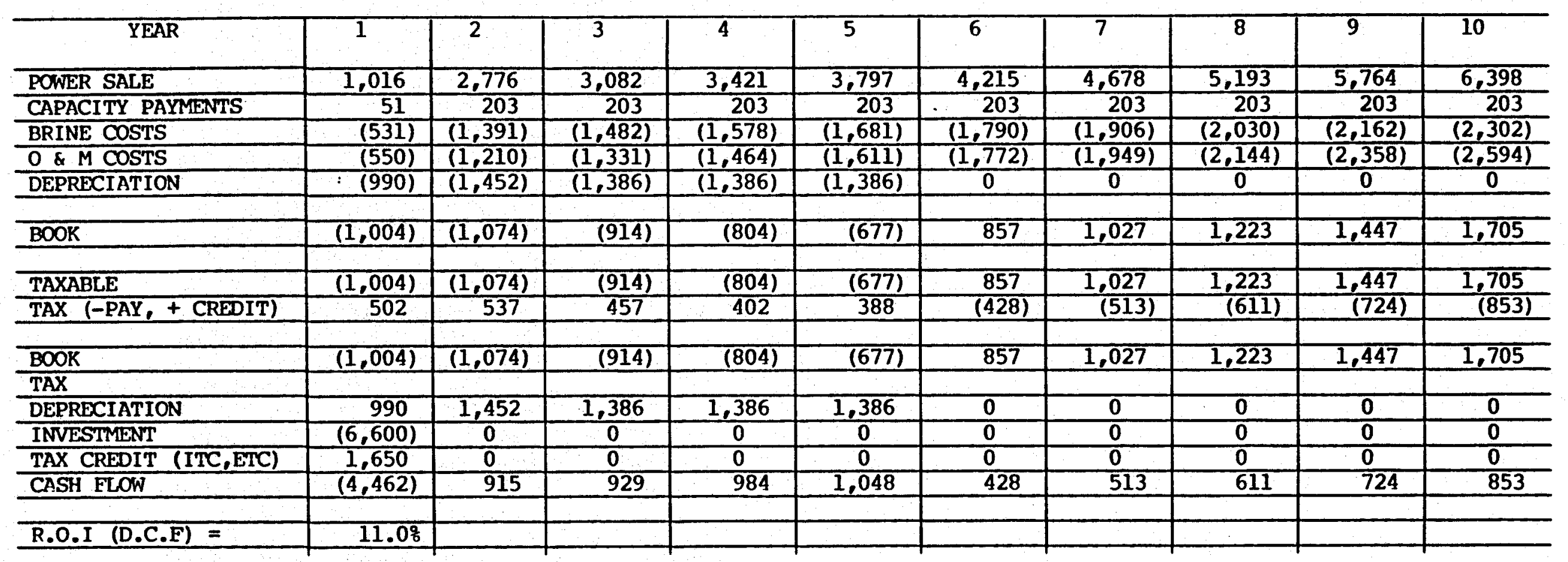

PART I STUDY - $\$ 6,000 K$ CAPITAL COST - BINARY 


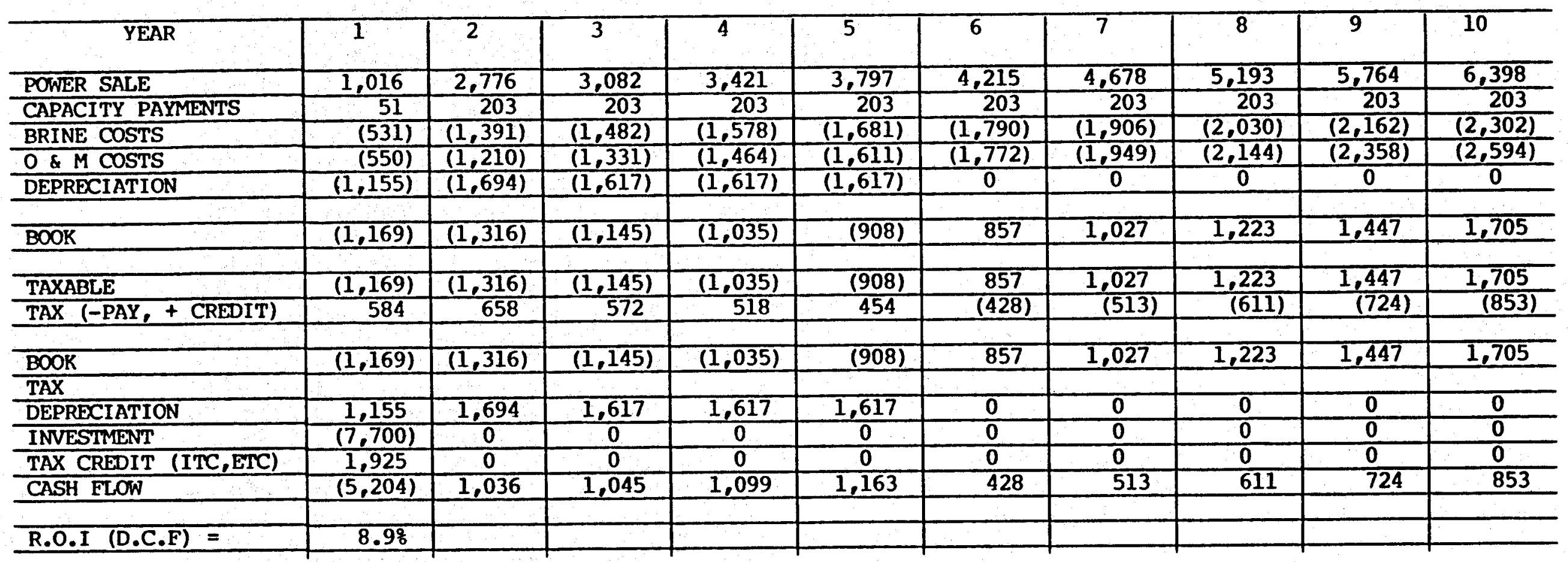

PART I STUDY - \$7,000K CAPITAL COST - BINARY 


\begin{tabular}{|c|c|c|c|c|c|c|c|c|c|c|}
\hline YEAR & 1 & 2 & 3 & 4 & 5 & 6 & 7 & 8 & 9 & 10 \\
\hline POWER SALE & 1,337 & 3,653 & 4,055 & 4,501 & 4,996 & 5,546 & 6,156 & 6,833 & 7,584 & 8,419 \\
\hline CAPACITY PAYMENTS & 67 & 267 & 267 & 267 & 267 & 267 & 267 & 267 & 267 & 267 \\
\hline BRINE COSTS & (531) & $(1,391)$ & $(1,481)$ & $(1,578)$ & $(1,680)$ & $(1,789)$ & $(1,906)$ & $(2,030)$ & $(2,162)$ & $(2,302)$ \\
\hline $0 \& M$ COSTS & (275) & $(605)$ & $(666)$ & (732) & $(805)$ & $(886)$ & $(974)$ & $(1.072)$ & $(1,179)$ & $(1,297)$ \\
\hline DEPRECIATION & $(825)$ & $(1,210)$ & $(1,155)$ & $(1,155)$ & $(1,155)$ & 0 & 0 & 0 & 0 & 0 \\
\hline BOOK & $(227)$ & 714 & 1,020 & 1,304 & 1,623 & 3,138 & 3,543 & 3,999 & 4.511 & 5,087 \\
\hline TAXABLE & (227) & 714 & 1,020 & 1,304 & 1,623 & 3,138 & 3,543 & 3,999 & 4,511 & 5,087 \\
\hline TAX (-PAY, + CREDIT) & 113 & $(357)$ & (510) & (652) & (811) & $(1,569)$ & $(1,771)$ & $(1,999)$ & $(2,256)$ & $(2,544)$ \\
\hline BOOK & (227) & 714 & 1,020 & 1,304 & 1,623 & 3,138 & 3,543 & 3,999 & 4.511 & 5,087 \\
\hline TAX & & & & & & & & & & \\
\hline DEPRECIATION & 825 & 1,210 & 1,155 & 1,155 & 1,155 & $\overline{0}$ & 0 & $\overline{0}$ & 0 & 0 \\
\hline INVESTMENT & $(5,500)$ & 0 & 0 & 0 & 0 & $\mathbf{0}$ & 0 & 0 & 0 & 0 \\
\hline TAX CREDIT (ITC, ETC) & 1,374 & 0 & 0 & 0 & 0 & 0 & 0 & 0 & 0 & 0 \\
\hline CASH FLOW & $(3,413)$ & 1,567 & 1,665 & 1,807 & 1,966 & 1,569 & 1,771 & 1,999 & 2,256 & 2,544 \\
\hline R.O.I (D.C.F) $=$ & $49.0 \%$ & & & & & & & & & \\
\hline
\end{tabular}

PART I STUDY - \$500K $0 \& M$ - Gravity Head 


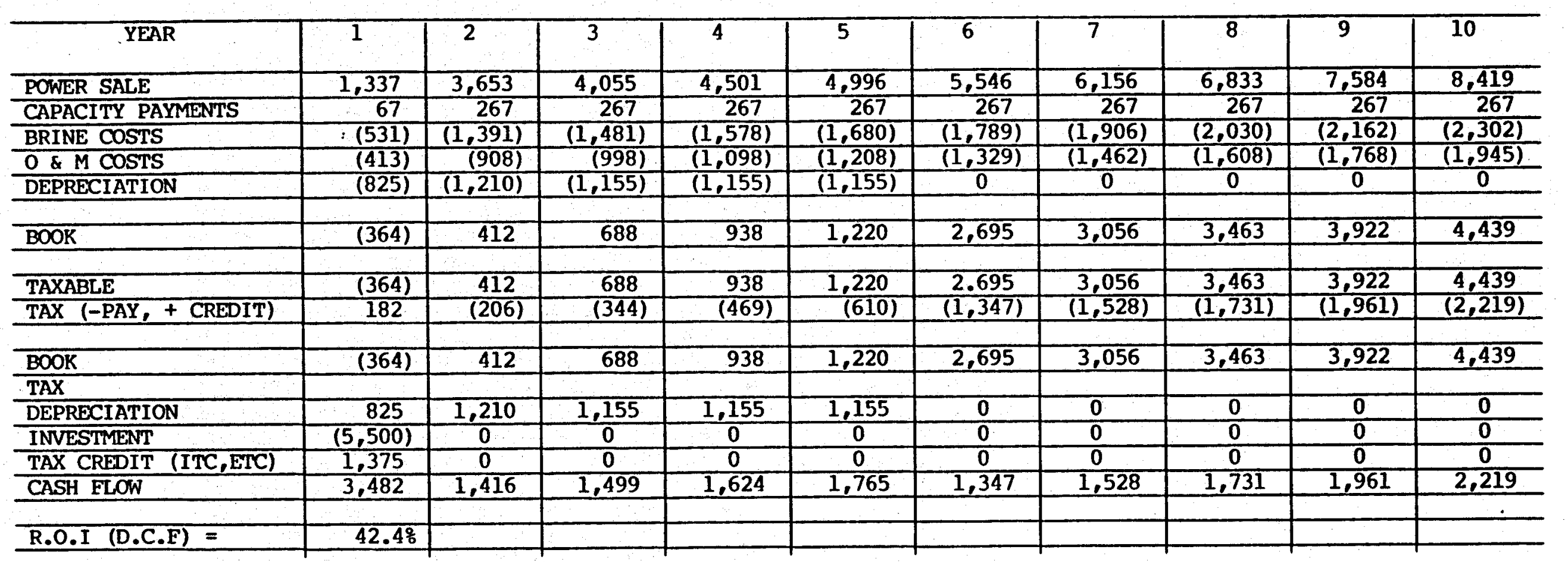

PART I STUDY --\$750K O \& M-- Gravity Head 


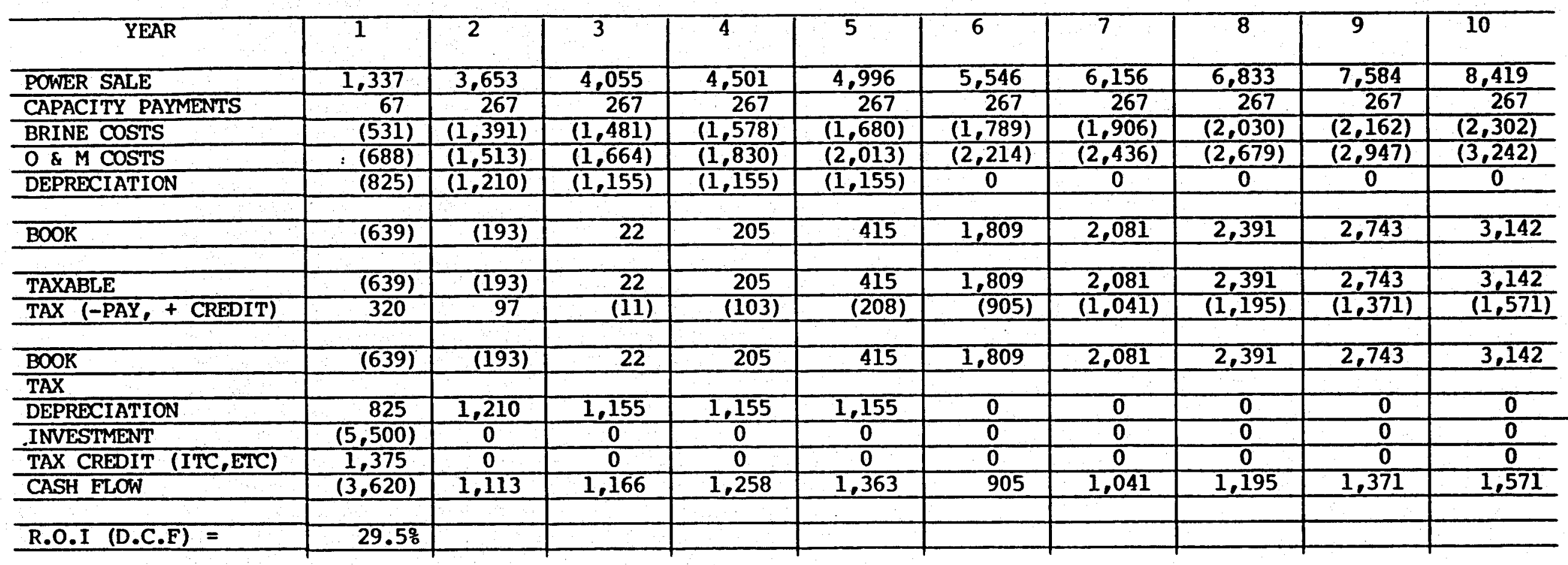

PART I STUDY - $\$ 1,250 K$ o \& M- Gravity Head 


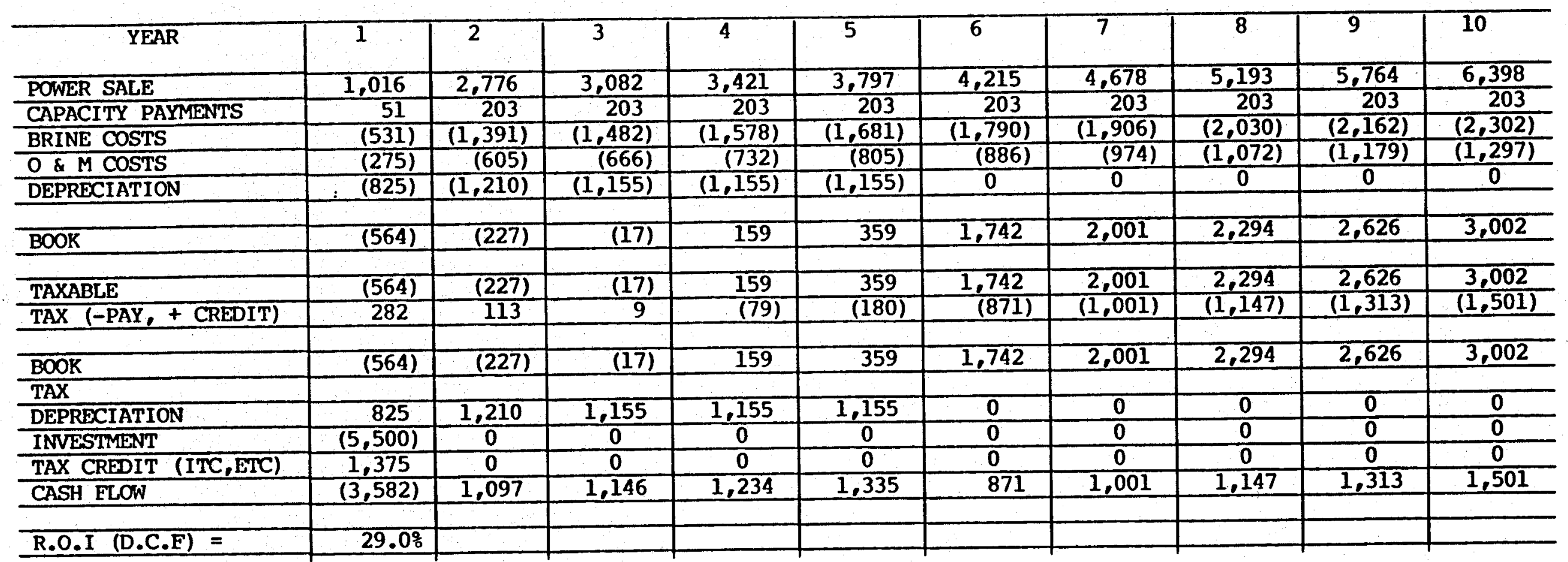

PART I STUDY - \$500 K O\&M - BINARY 


\begin{tabular}{|c|c|c|c|c|c|c|c|c|c|c|}
\hline YEAR & 1 & 2 & 3 & 4 & 5 & 6 & 7 & 8 & 9 & 10 \\
\hline POWER SALE & 1,016 & 2,776 & 3,082 & 3,421 & 3,797 & 4,215 & 4,678 & 5,193 & 5,764 & 6,398 \\
\hline CAPACITY PAYMENTS & 51 & 203 & 203 & 203 & 203 & 203 & 203 & 203 & 203 & 203 \\
\hline BRINE COSTS & (531) & $(1,391)$ & $(1,482)$ & $(1,578)$ & $(1,681)$ & $(1,790)$ & $(1,906)$ & $(2,030)$ & $(2,162)$ & $(2,302)$ \\
\hline $0 \& M$ COSTS & (413) & (908) & (998) & $(1,098)$ & $(1,208)$ & $(1,329)$ & $(1,462)$ & $(1,608)$ & $(1,768)$ & $(1,945)$ \\
\hline DEPRECIATION & $(825)$ & $(1,210)$ & $(1,155)$ & $(1,155)$ & $(1,155)$ & 0 & 0 & 0 & 0 & 0 \\
\hline & & & & & & & & & & \\
\hline BOOK & (701) & (529) & $(350)$ & $(207)$ & $(43)$ & 1,299 & 1,514 & 1,758 & 2,037 & 2,354 \\
\hline TAXABLE & (701) & $(529)$ & $(350)$ & & & & & & 2.037 & 2,354 \\
\hline TAX (-PAY, + CREDIT) & (351) & 265 & $\frac{1000}{175}$ & $\frac{(207)}{104}$ & $\frac{(43)}{22}$ & $\frac{1,299}{(650)}$ & $\frac{1,514}{(757)}$ & $\frac{1,158}{(879)}$ & $\frac{2,031}{(1,018)}$ & $\frac{2,304}{(1,177)}$ \\
\hline & & & & & & & & & & \\
\hline$\frac{B O O K}{\text { TAX }}$ & (701) & (529) & $(350)$ & (207) & (43) & 1,299 & 1,514 & 1,758 & 2,037 & 2,354 \\
\hline & & & & & & & & & & \\
\hline $\begin{array}{l}\text { DEPRECIATION } \\
\text { INVESTMENT }\end{array}$ & 825 & 1,210 & 1,155 & 1.155 & 1,155 & 0 & 0 & 0 & 0 & 0 \\
\hline $\begin{array}{l}\text { INVESTMENT } \\
\text { TAX CREDIT (ITC,ETC) }\end{array}$ & $(5,500)$ & 0 & 0 & 0 & 0 & 0 & 0 & 0 & 0 & 0 \\
\hline $\begin{array}{l}\text { TAX CREDIT (ITC,ETC) } \\
\text { CASH ELOW }\end{array}$ & 1,375 & 0 & 0 & 0 & 0 & 0 & 0 & $\frac{0}{070}$ & 0 & 0 \\
\hline & $(3,651)$ & 945 & 980 & 1,051 & 1,133 & 650 & 757 & 879 & 1,018 & \\
\hline R.O.I (D.C.F) $=$ & $21.8 \%$ & & & & & & & & & \\
\hline
\end{tabular}

PART I STUDY - \$750K O\&M - BINARY 


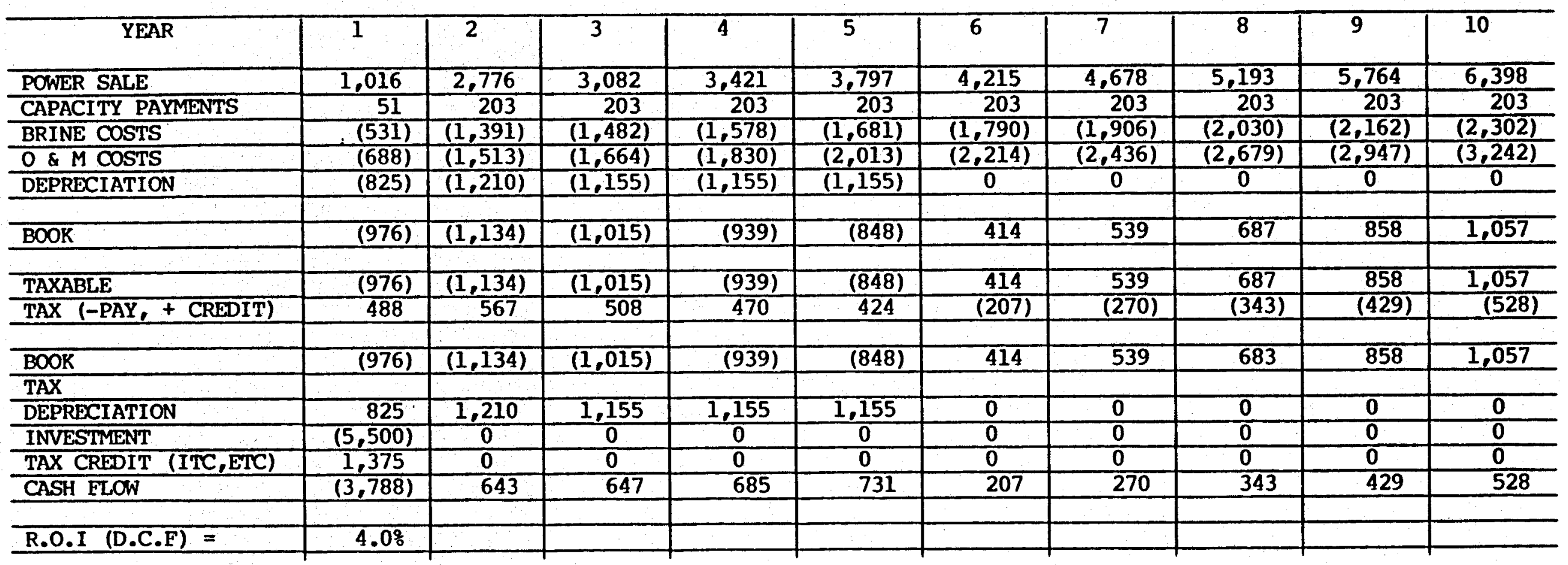

PART I STUDY - $\$ 1,250 \mathrm{~K}$ O\&M - BINARY 


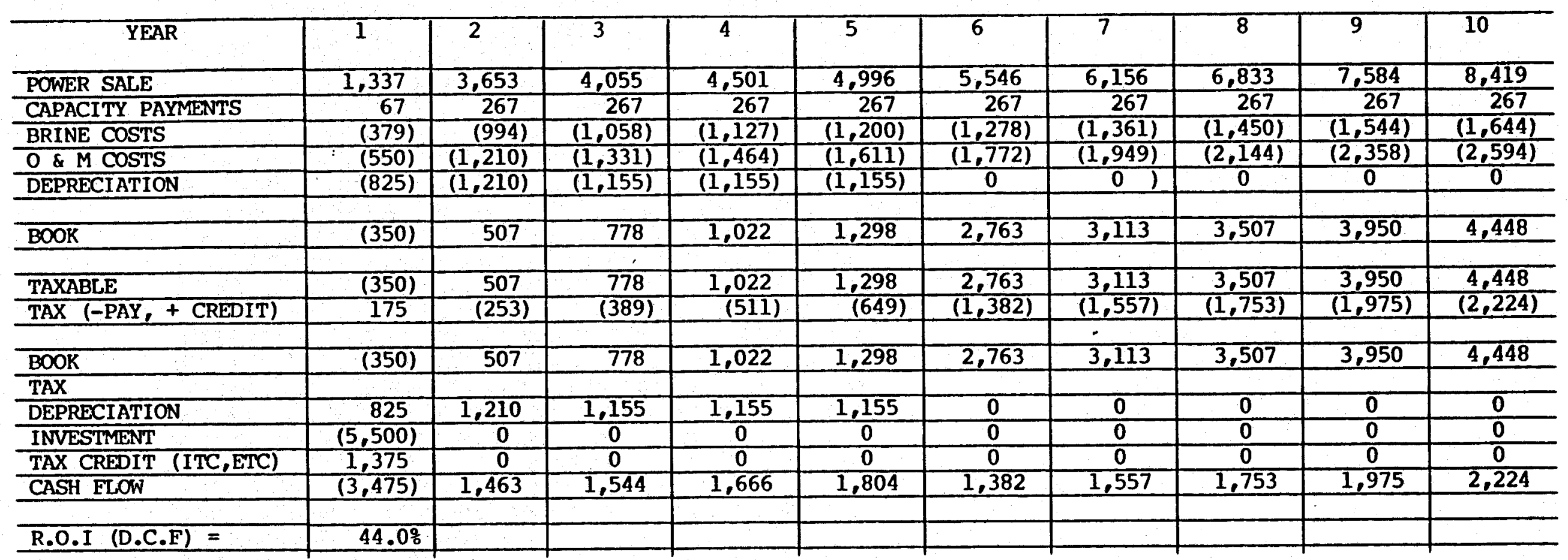

PART I STUDY - 21.6338c/1000 lb. BRINE - GRAVITY HEAD 


\begin{tabular}{|c|c|c|c|c|c|c|c|c|c|c|}
\hline YEAR & 1 & 2 & 3 & 4 & 5 & $\overline{6}$ & 7 & 8 & 9 & 10 \\
\hline POWER SALE & 1,337 & 3,653 & 4,055 & 4,501 & 4,996 & 5,546 & 6,156 & 6,833 & 7,584 & 8,419 \\
\hline CAPACITY PAYMENTS & 67 & 267 & 267 & 267 & 267 & 267 & 267 & 267 & 267 & 267 \\
\hline BRINE COSTS & $(606)$ & $(1,590)$ & $(1,693)$ & $(1,803)$ & $(1,920)$ & $(2,045)$ & $(2,178)$ & $(2,320)$ & $(2,470)$ & $(2,631)$ \\
\hline O\&M COSTS & $\therefore \quad(550)$ & $(1,210)$ & $(1,331)$ & $(1,464)$ & $(1,611)$ & $(1,772)$ & $(1,949)$ & $(2,144)$ & $(2,358)$ & $(2,594)$ \\
\hline DEPRECIATION & $(825)$ & $(1,210)$ & $(1,155)$ & $(1,155)$ & $(1,155)$ & 0 & 0 & 0 & 0 & 0 \\
\hline BOOK & $(578)$ & (89) & 143 & 346 & 578 & 1,996 & 2,296 & 2,637 & 3,023 & 3,461 \\
\hline & & & & & & & & & & \\
\hline TAXABLE & $(578)$ & (89) & 143 & 346 & 578 & 1,996 & 2,296 & 2,637 & 3,023 & $\frac{3,461}{7211}$ \\
\hline TAX (-PAY, + CREDIT) & 289 & 45 & (72) & $(173)$ & $(289)$ & (998) & $(1,148)$ & $(1,318)$ & $(1,512)$ & $(1,731)$ \\
\hline BOOK & (578) & $(89)$ & 143 & 346 & 578 & 1,996 & 2,296 & 2,637 & 3,023 & 3,461 \\
\hline TAX & & & & & & & & & & \\
\hline DEPRECIATION & 825 & 1,210 & 1,155 & 1,155 & $(1,155)$ & $\overline{0}$ & 0 & 0 & 0 & 0 \\
\hline INVESTMENT & $(5,500)$ & 0 & 0 & 0 & 0 & 0 & 0 & $\mathbf{0}$ & 0 & 0 \\
\hline TAX CREDIT (ITC,ETC) & 1.375 & 0 & 0 & 0 & 0 & 0 & 0 & 0 & 0 & 0 \\
\hline CASH FLOW & $(3,589)$ & 1,165 & 1,227 & 1,328 & 1,444 & 998 & 1,148 & 1,318 & 1,512 & 1,731 \\
\hline R.O.I (D.C.F) $=$ & $32.0 \%$ & & & & & & & & & \\
\hline
\end{tabular}

PART I STUDY - 34.6141c/1000 1b. - brine - Gravity Head 


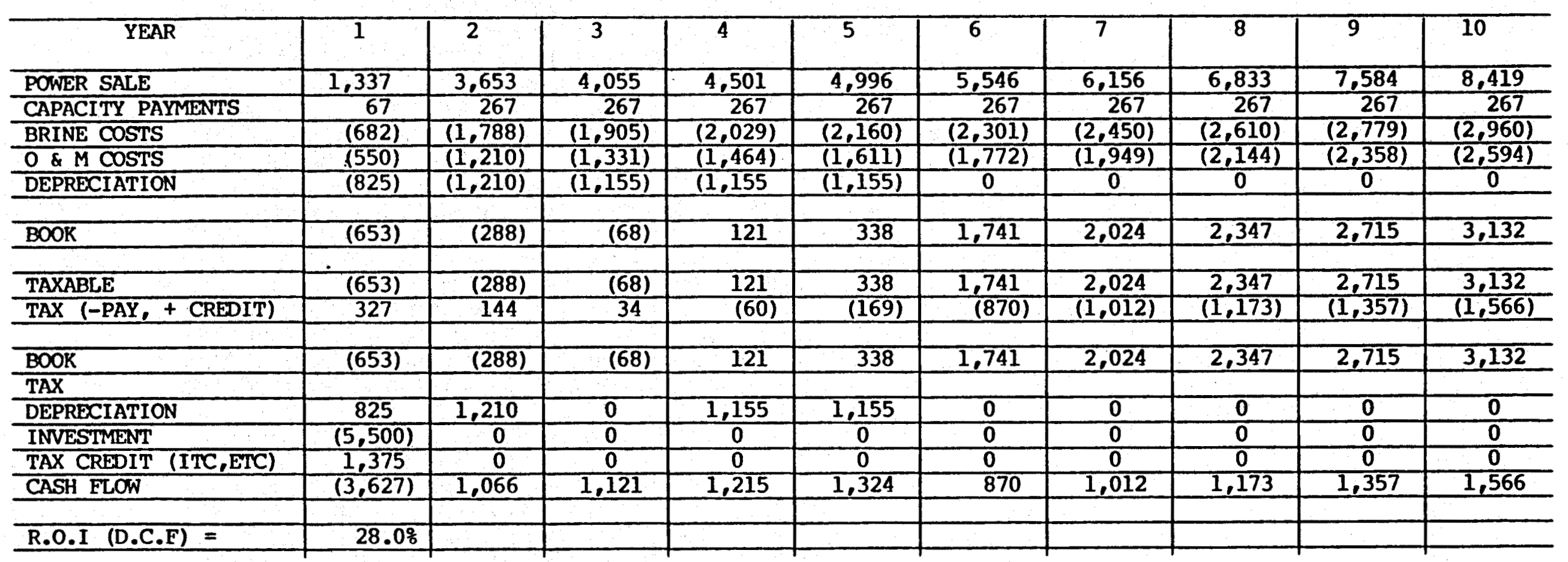

PART I STUDY - 38.9408c/1000 1b. brine - Gravity Head 


\begin{tabular}{|c|c|c|c|c|c|c|c|c|c|c|}
\hline YEAR & 1 & 2 & 3 & 4 & 5 & $\overline{6}$ & 7 & 8 & 9 & 10 \\
\hline POWER SALE & 1,016 & 2,776 & 3,082 & 3,421 & 3,797 & 4.215 & 4,678 & 5,193 & 5,764 & 6,398 \\
\hline CAPACITY PAYMENTS & 51 & 203 & 203 & 203 & 203 & 203 & 203 & 203 & 203 & 203 \\
\hline BRINE COSTS & (379) & (994) & $(1,058)$ & $(1,127)$ & $(1,200)$ & $(1,278)$ & $(1,362)$ & $(1,450)$ & $(1,544)$ & $(1,645)$ \\
\hline $0 \& M \operatorname{COSTS}$ & $(550)$ & $(1,210)$ & $(1,331)$ & $(1,464)$ & $(1,611)$ & $(1,772)$ & $(1,949)$ & $(2,144)$ & $(2,358)$ & $(2,594)$ \\
\hline DEPRECIATION & $(825)$ & $(1,210)$ & $(1,155)$ & $(1,155)$ & $(1,155)$ & 0 & 0 & 0 & 0 & 0 \\
\hline BOOK & $(687)$ & $(434)$ & (259) & $(122)$ & 34 & 1,368 & 1,571 & 1,803 & 2,065 & 2,363 \\
\hline & & & & & & & & & & \\
\hline $\begin{array}{l}\text { TAXABLE } \\
\text { TAX } 1-\text { DAY }+ \text { CRFDTT }\end{array}$ & $(687)$ & $(434)$ & 130 & $(122)$ & 34 & 1,368 & 1,571 & 1,803 & 2,065 & 2,363 \\
\hline TAX (-PAY, + CREDIT) & 344 & 217 & 130 & 61 & (17) & $(684)$ & $(786)$ & 901 & $(1,033)$ & \\
\hline BOOK & $(687)$ & (434) & (259) & (122) & 34 & 1,368 & 1,571 & 1,806 & 2,065 & 2,363 \\
\hline TAX & & & & & & & & & & \\
\hline DEPRECIATION & 825 & 1,210 & 1,155 & 1,155 & 1,155 & 0 & $\overline{0}$ & $\overline{0}$ & 0 & 0 \\
\hline INVESTMENT & $(5,500)$ & 0 & 0 & 0 & 0 & 0 & 0 & 0 & 0 & 0 \\
\hline TAX CREDIT (ITC,ETC) & 1.375 & 0 & 0 & 0 & 0 & 0 & 0 & 0 & 0 & 0 \\
\hline CASH FLOW & $(3,644)$ & 993 & & 1,094 & 1,172 & 684 & 786 & 901 & 1,033 & 1,182 \\
\hline & & & & & & & & & & \\
\hline R.O.I (D.C.F) $=$ & $23 \%$ & & & & & & & & & \\
\hline
\end{tabular}




\begin{tabular}{|c|c|c|c|c|c|c|c|c|c|c|}
\hline YEAR & 1 & 2 & 3 & 4 & 5 & 6 & 7 & 8 & 9 & 10 \\
\hline POWER SALE & 1,016 & 2,776 & 3,082 & 3,421 & 3,797 & 4,215 & 4.678 & 5,193 & 5.764 & 5,698 \\
\hline CAPACITY PAYMENTS & 51 & 203 & 203 & 203 & 203 & 203 & 203 & 203 & 203 & 203 \\
\hline BRINE COSTS & $(607)$ & $(1,590)$ & $(1,693)$ & $(1,803)$ & $(1,921)$ & $(2,045)$ & $(2,178)$ & $(2,320)$ & $(2,471)$ & $(2,631)$ \\
\hline O\& M COSTS & $(550)$ & $(1,210)$ & $(1.331)$ & $(1,464)$ & $(1,611)$ & $(1,772)$ & $(1,949)$ & $(2,144)$ & $(2,358)$ & $(2,594)$ \\
\hline DEPRECIATION & $(825)$ & $(1,210)$ & $(1,155)$ & $(1,155)$ & $(1,155)$ & 0 & 0 & 0 & 0 & 0 \\
\hline $\mathrm{BOOK}$ & (915) & $(1,030)$ & $(894)$ & (799) & (686) & 601 & 754 & 933 & 1,139 & 1,376 \\
\hline & & & & & & & & & & \\
\hline TAXABLE & (915) & $(1,030)$ & (894) & (799) & $(686)$ & 601 & 754 & 933 & 1,139 & 1,376 \\
\hline TAX $(-\mathrm{PAY},+$ CREDIT $)$ & 457 & 515 & 447 & 399 & 343 & $(300)$ & $(377)$ & $(466)$ & (569) & $(688)$ \\
\hline $\mathrm{BOOK}$ & (915) & $(1,030)$ & $(894)$ & (799) & $(686)$ & 601 & $\overline{754}$ & 933 & 1,139 & 1,376 \\
\hline TAX & & & & & & & & & & \\
\hline DEPRECIATION & 825 & 1,210 & 1,155 & 1,155 & 1,155 & $\overline{0}$ & $\overline{0}$ & $\overline{0}$ & $\overline{0}$ & $\begin{array}{c}\mathbf{0} \\
\end{array}$ \\
\hline INVESTMENT & $(5,500)$ & 0 & 0 & 0 & 0 & 0 & 0 & $\overline{0}$ & 0 & $\overline{0}$ \\
\hline TAX CREDIT (ITC,ETC) & 1.375 & 0 & $\overline{0}$ & 0 & $\overline{0}$ & 0 & 0 & 0 & 0 & 0 \\
\hline CASH FLOW & 3,757 & 695 & 708 & 756 & 812 & 300 & 377 & 466 & 569 & 688 \\
\hline R.0.I (D.C.F) $=$ & 8.49 & & & & & & & & & \\
\hline
\end{tabular}




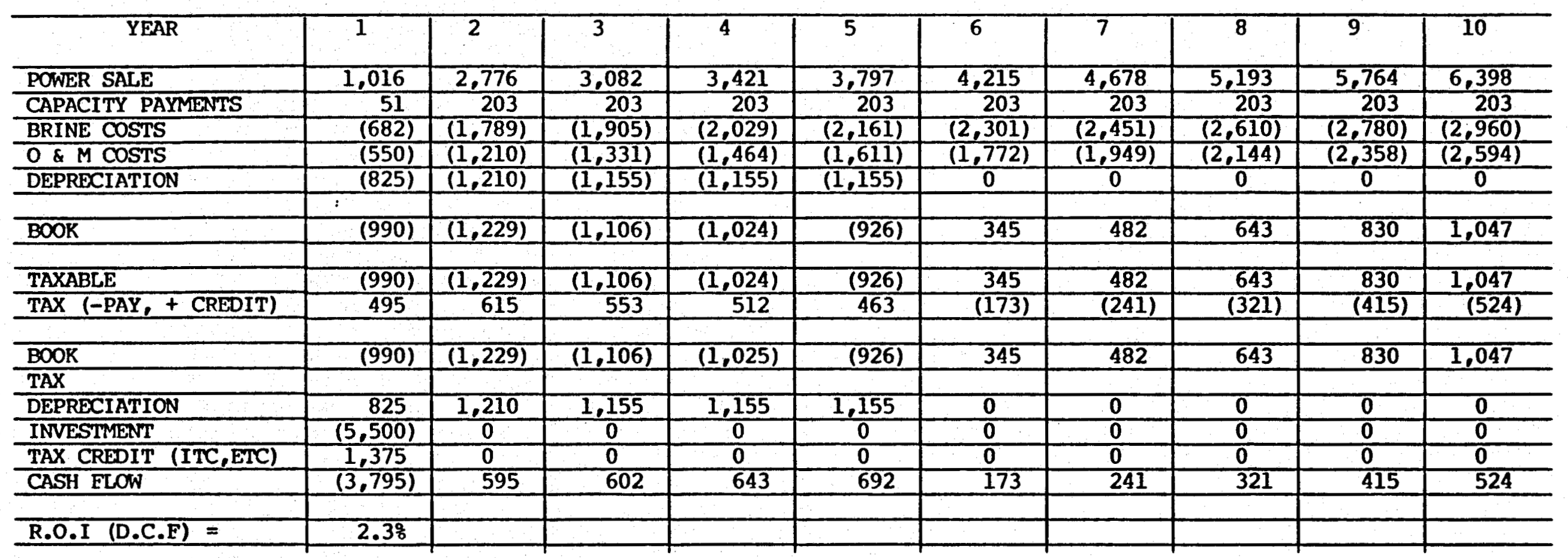

PART I STUDY - 38.9408c/1000 1b BRINE - BINARY 


\begin{tabular}{|c|c|c|c|c|c|c|c|c|c|c|}
\hline YEAR & 1 & $\overline{2}$ & 3 & 4 & 5 & 6 & 7 & 8 & 9 & 10 \\
\hline POWER SALE & 1,851 & 14,498 & 20.275 & 22,505 & 24,980 & 27.728 & 30,778 & 34,164 & 37,922 & 42,093 \\
\hline CAPACITY PAYMENTS & 67 & 1.100 & 1.599 & 1.599 & 1,599 & 1.599 & 1,599 & 1,599 & 1,599 & 1,599 \\
\hline BRINE COSTS & $(210)$ & $(1.577)$ & $(2,116)$ & $(2,254)$ & $(2,400)$ & $(2,556)$ & $(2,723)$ & $(2,900)$ & $(3,088)$ & $(3,289)$ \\
\hline $0 \& M$ COSTS & $:(619)$ & $(2,193)$ & $(2,662)$ & $(2,928)$ & $(3,221)$ & $(3,543)$ & $(3,897)$ & $(4,287)$ & $(4,716)$ & $(5,187)$ \\
\hline DEPRECIATION & $(4,049)$ & $(8,841)$ & $(9,925)$ & $(9,731)$ & $(9,731)$ & $(4,063)$ & 0 & 0 & 0 & 0 \\
\hline $\mathrm{BOOK}$ & $(2,960)$ & 2,987 & 7,170 & 9,190 & 11,226 & 19,165 & 25,757 & 28.576 & 31,717 & 35,216 \\
\hline TAXABLE & $(2,960)$ & 2,987 & 7,170 & 9,190 & 11,226 & 19.165 & 25.757 & 28,576 & 31.717 & 35.216 \\
\hline TAX (-PAY,+ CREDIT) & 1,480 & $(1,493)$ & $(3,585)$ & $(4,595)$ & $(5,613)$ & $(9,582)$ & $(12,879)$ & $(14,288)$ & $(15,858)$ & $(17,608)$ \\
\hline$\overline{\mathrm{BOOK}}$ & $(2,960)$ & 2,987 & 7,170 & 9,190 & 11,226 & 19,165 & 25,757 & 28,576 & 31,717 & 35,216 \\
\hline TAX & & & & & & & & & & \\
\hline DEPRECIATION & 4,049 & 8,841 & 9,925 & 9,731 & 9,731 & 4,063 & 0 & 0 & 0 & $\mathbf{0}$ \\
\hline INVESTMENT & $(31,493)$ & $(14,847)$ & 0 & 0 & 0 & 0 & $\mathbf{0}$ & $\mathbf{0}$ & 0 & $\mathbf{0}$ \\
\hline TAX CREDIT (ITC,ETC) & 7,873 & 3,712 & 0 & 0 & 0 & 0 & 0 & 0 & 0 & 0 \\
\hline CASH FLOW & $(21,050)$ & $(801)$ & 13,510 & 14,326 & 15,345 & 13,645 & 12,879 & 14,288 & 15,858 & 17,608 \\
\hline R.0.I (D.C.F) $=$ & $43.23 \%$ & & & & & & & & & \\
\hline
\end{tabular}

PART II STUDY - FIVE-UNIT GRAVITY HEAD WITH 8.6535c/1000 1b. BRINE COST - WELL COST INCLUDED 


\begin{tabular}{|c|c|c|c|c|c|c|c|c|c|c|}
\hline YEAR & 1 & 2 & 3 & 4 & 5 & 6 & 7 & $\overline{8}$ & 9 & 10 \\
\hline POWER SALE & 1,851 & 14,498 & 20,275 & 22,505 & 24,980 & 27,728 & 30,778 & 34,164 & 37,922 & 42,093 \\
\hline CAPACITY PAYMENTS & 67 & 1,100 & 1,599 & 1,599 & 1,599 & 1,599 & 1,599 & 1,599 & 1,599 & 1,599 \\
\hline BRINE COSTS & $(420)$ & $(3,155)$ & $(4,233)$ & $(4,508)$ & $(4,801)$ & $(5,113)$ & $(5,445)$ & $(5,799)$ & $(6,176)$ & $(6,577)$ \\
\hline $0 \& M$ COSTS & (619) & $(2,193)$ & $(2,662)$ & $(2,928)$ & $(3,221)$ & $(3,543)$ & $(3,897)$ & $(4,287)$ & $(4,716)$ & $(5,187)$ \\
\hline DEPRECIATION & $(4,049)$ & $(8,841)$ & $(9,925)$ & $(9,731)$ & $(9,731)$ & $(4,063)$ & 0 & 0 & 0 & 0 \\
\hline $\mathrm{BOOK}$ & $(3,170)$ & 1,409 & 5,054 & 6,936 & 8,826 & 16,609 & 23,035 & 25,676 & 28,629 & 31,927 \\
\hline TAXABLE & $(3,170)$ & 1,409 & 5,054 & 6,936 & 8,826 & 16,609 & 23,035 & 25,676 & 28,629 & 31,927 \\
\hline TAX $\left(-\mathrm{PAY}_{1}+{ }^{+}\right.$CREDIT $)$ & 1,585 & $(705)$ & $(2,527)$ & $(3,468)$ & $(4,413)$ & $(8,304)$ & $(11,517)$ & $(12,838)$ & $(14,314)$ & $(15,964)$ \\
\hline $\mathrm{BOOK}$ & $(3,170)$ & 1,409 & 5.054 & 6,936 & 8,826 & 16,609 & 23.035 & 25,676 & 28,629 & 31,927 \\
\hline TAX & & & & & & & & & & \\
\hline DEPRECIATION & 4,049 & 8,841 & 9,925 & 9,731 & 9,731 & 4,063 & 0 & $\mathbf{0}$ & 0 & 0 \\
\hline INVESTMENT & $(31,493)$ & $(14,847)$ & 0 & 0 & 0 & 0 & 0 & 0 & 0 & 0 \\
\hline TAX CREDIT (ITC, ETC) & 7,873 & 3,712 & 0 & 0 & 0 & 0 & 0 & 0 & 0 & $\mathbf{0}$ \\
\hline CASH FLOW & $(21,115)$ & $(1,590)$ & 12,452 & 13,200 & 14,144 & 12,367 & 11,517 & 12,838 & 14,314 & 15,964 \\
\hline R.O.I (D.C.F) $=$ & $38.95 \%$ & & & & & & & & & \\
\hline
\end{tabular}

PART II STUDY - FIVE-UNIT GRAVITY HEAD WITH 17.247c/1000 Ib BRINE COST - WELL COST INCLUDED 


\begin{tabular}{|c|c|c|c|c|c|c|c|c|c|c|}
\hline YEAR & 1 & 2 & 3 & 4 & 5 & 6 & 7 & 8 & 9 & 10 \\
\hline POWER SALE & 1,407 & 11,019 & 15,409 & 17,104 & 18,985 & 21.073 & 23,391 & 25,965 & 28,821 & 31,991 \\
\hline CAPACITY PAYMENTS & 51 & 836 & 1.215 & 1,215 & 1,215 & 1,215 & 1,215 & 1,215 & 1,215 & 1.215 \\
\hline BRINE COSTS & $(210)$ & $(1,578)$ & $(2,117)$ & $(2,254)$ & $(2,401)$ & $(2,557)$ & $(2,723)$ & $(2,900)$ & $(3,089)$ & $(3,289)$ \\
\hline O\& M COSTS & $(619)$ & $(2,193)$ & $(2,662)$ & $(2,928)$ & $(3,221)$ & $(3,543)$ & $(3,897)$ & $(4,287)$ & $(4,716)$ & $(5,187)$ \\
\hline DEPRECIATION & $(4,107)$ & $(8,966)$ & $(10,066)$ & $(9,869)$ & $(9,869)$ & $(4,120)$ & 0 & 0 & 0 & 0 \\
\hline & & & & & & & 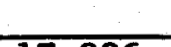 & & & \\
\hline $\mathrm{BOOK}$ & $(3,477)$ & $(882)$ & 1,780 & 3,267 & 4.709 & 12,068 & 17.986 & 19,993 & 22,231 & 24,729 \\
\hline TAXABLE & $(3,477)$ & $(882)$ & 1,780 & 3,267 & 4,709 & 12,068 & 17,986 & 19,993 & 22,231 & 24,729 \\
\hline TAX $\left(-\mathrm{PAY}_{8}+\right.$ CREDIT $)$ & 1,736 & 441 & $(890)$ & $(1,634)$ & $(3,255)$ & $(6,034)$ & $(8,993)$ & $(9,996)$ & $(11,116)$ & $(12,365)$ \\
\hline BOOK & $(3,477)$ & $(882)$ & 1,780 & 3,267 & 4,709 & 12,068 & 17,986 & 19,993 & 22,231 & 24,729 \\
\hline $\operatorname{TAX}$ & & & & & & & & & & \\
\hline DEPRECIATION & 4,107 & 8,966 & 10,066 & 9,869 & 9,869 & 4,120 & 0 & 0 & $\mathbf{0}$ & $\mathbf{0}$ \\
\hline INVESTMENT & $(31,940)$ & $(15,057)$ & 0 & 0 & 0 & 0 & 0 & 0 & 0 & 0 \\
\hline TAX CREDIT (ITC,ETC) & 7,985 & 3,764 & 0 & 0 & 0 & 0 & 0 & 0 & 0 & 0 \\
\hline CASH FLOW & $(21,587)$ & $(2,768)$ & 10,955 & 11,503 & 12,224 & 10,154 & 8,993 & 9,996 & 11,116 & 12,365 \\
\hline R.O.I (D.C.F) $=$ & $31.34 \%$ & & & & & & & & & \\
\hline
\end{tabular}

PART II STUDY - FIVE-UNIT BINARY WITH 8.6535c/1000 Ib BRINE COST - WELL COST INCLUDED. 


\begin{tabular}{|c|c|c|c|c|c|c|c|c|c|c|}
\hline YEAR & 1 & 2 & 3 & 4 & 5 & 6 & 7 & 8 & 9 & 10 \\
\hline POWER SALE & 1,407 & 11,019 & 15,409 & 17,104 & 18,985 & 21,073 & 23,391 & 25,965 & 28,821 & 31,991 \\
\hline CAPACITY PAYMENTS & 51 & 836 & 1,215 & 1,215 & 1,215 & 1,215 & 1,215 & 1,215 & 1,215 & 1,215 \\
\hline BRINE COSTS & $(420)$ & $(3,155)$ & $(4,233)$ & $(4,508)$ & $(4,802)$ & $(5,114)$ & $(5,446)$ & $(5,800)$ & $(6,177)$ & $(6,579)$ \\
\hline $0 \& \quad$ COSTS & $:(619)$ & $(2,193)$ & $(2,662)$ & $(2,928)$ & $(3,221)$ & $(3,543)$ & $(3,897)$ & $(4,287)$ & $(4,716)$ & $(5,187)$ \\
\hline DEPRECIATION & $(4,107)$ & $(8,966)$ & $(10,066)$ & $(9,869)$ & $(9,869)$ & $(4,120)$ & 0 & 0 & 0 & 0 \\
\hline BOOK & $(3,687)$ & $(2,460)$ & $(337)$ & 1,013 & 2,308 & 9,512 & 15,263 & 17,093 & 19,143 & 21,440 \\
\hline & & & & & & & & & & \\
\hline TAX $(-\mathrm{PAY},+$ CREDIT $)$ & 1,844 & 1,230 & 169 & $(506)$ & $(1,154)$ & $(4,756)$ & $(7.632)$ & $(8,546)$ & $(9,571)$ & $(10,720)$ \\
\hline $\mathrm{BOOK}$ & $(3,687)$ & $(2,460)$ & (337) & 1,013 & 2,308 & 9,512 & 15,263 & 17.093 & 19,143 & 21,440 \\
\hline TAX & & & & & & & & 11,000 & 10.143 & 21.440 \\
\hline DEPRECIATION & 4,107 & 8,966 & 10,066 & 9.869 & 9,869 & 4,120 & 0 & 0 & $\overline{0}$ & $\overline{0}$ \\
\hline INVESTMENT & $(31,940)$ & $(15,057)$ & 0 & 0 & 0 & 0 & 0 & 0 & 0 & $\mathbf{0}$ \\
\hline TAX CREDIT (ITC,ETC) & 7,985 & 3,764 & 0 & 0 & 0 & 0 & 0 & 0 & 0 & 0 \\
\hline CASH FLOW & $(21,692)$ & $(3,557)$ & 9.897 & 10,376 & 11,023 & 8,876 & 7,632 & 8,546 & 9,571 & 10,720 \\
\hline
\end{tabular}

PART II STUDY - FIVE-UNIT BINARY WITH 17.247c/1000 lb BRINE COST - WELL COST INCLUDED 


\begin{tabular}{|c|c|c|c|c|c|c|c|c|c|c|}
\hline YEAR & 1 & 2 & 3 & 4 & 5 & 6 & 7 & 8 & 9 & 10 \\
\hline POWER SALE & 1,337 & 3,653 & 4,055 & 4,501 & 4,996 & 5,546 & 6,156 & 6,833 & 7,584 & 8,419 \\
\hline CAPACITY PAYMENTS & 67 & 267 & 267 & 267 & 267 & 267 & 267 & 267 & 267 & 267 \\
\hline BRINE COSTS & (531) & $(1,391)$ & $(1,481)$ & $(1,578)$ & $(1,680)$ & $(1,789)$ & $(1,906)$ & $(2,030)$ & $(2,162)$ & $(2,302)$ \\
\hline$O \& M$ COSTS & (550) & $(1,210)$ & $(1,331)$ & $(1,464)$ & $(1,611)$ & $(1,772)$ & $(1,949)$ & $(2,144)$ & $(2,358)$ & $(2,594)$ \\
\hline DEPRECIATION & $(1,030)$ & $(1,511)$ & $(1,442)$ & $(1,442)$ & $(1,442)$ & 0 & 0 & 0 & 0 & 0 \\
\hline $\mathrm{BOOK}$ & (707) & (191) & 68 & 285 & 531 & 2,252 & 2,569 & 2,927 & 3,332 & 3,790 \\
\hline$\frac{\text { TAXABLE }}{\text { TAX (-PAY, + CREDIT) }}$ & $\frac{(707)}{353}$ & $\frac{(191)}{96}$ & $\frac{68}{(34)}$ & $\frac{285}{(142)}$ & $\frac{531}{(265)}$ & $\frac{2,252}{(1,126)}$ & $\frac{2,569}{(1,284)}$ & $\frac{2,927}{(1,463)}$ & $\frac{3,332}{(1,666)}$ & $\frac{3,790}{(1,895)}$ \\
\hline $\mathrm{BOOK}$ & (707) & (191) & 68 & 285 & 531 & 2,252 & 2,569 & 2,927 & 3,332 & 3,790 \\
\hline TAX & & & & & & & & & & \\
\hline DEPRECIATION & 1,030 & 1,511 & 1,442 & 1,442 & 1,442 & $\overline{0}$ & 0 & $\overline{0}$ & $\overline{0}$ & $\overline{0}$ \\
\hline INVESTMENT & $(6,866)$ & 0 & 0 & 0 & 0 & 0 & 0 & 0 & 0 & 0 \\
\hline TAX CREDIT (ITC,ETC) & 1,717 & 0 & 0 & 0 & 0 & 0 & 0 & 0 & 0 & 0 \\
\hline CASH FLOW & $(4,473)$ & 1,415 & 1,476 & 1,584 & 1,707 & 1,126 & 1,284 & 1,463 & 1,666 & 1,895 \\
\hline
\end{tabular}

PART II STUDY - SINGLE-UNIT GRAVITY HEAD WITH $30.2873 \mathrm{c} / 1000$ Ib BRINE COST - WELL COST EXCLUDED 


\begin{tabular}{|c|c|c|c|c|c|c|c|c|c|c|}
\hline YEAR & 1 & 2 & 3 & 4 & 5 & $\overline{6}$ & 7 & 8 & 9 & 10 \\
\hline POWER SALE & 1,337 & 3,653 & 4,055 & 4,501 & 4,996 & 5,546 & 6,156 & 6,833 & 7.584 & 8,419 \\
\hline CAPACITY PAYMENTS & 67 & 267 & 267 & 267 & 267 & 267 & 267 & 267 & 267 & 267 \\
\hline BRINE COSTS & $(682)$ & $(1,788)$ & $(1,905)$ & $(2,029)$ & $(2,160)$ & $(2,301)$ & $(2,450)$ & $(2,610)$ & $(2,779)$ & $(2,960)$ \\
\hline$O \& M$ COSTS & $(550)$ & $(1,210)$ & $(1,331)$ & $(1,464)$ & $(1,611)$ & $(1,772)$ & $(1,949)$ & $(2,144)$ & $(2,358)$ & $(2,594)$ \\
\hline DEPRECIATION & $(1,030)$ & $(1,511)$ & $(1,442)$ & $(1,442)$ & $(1,442)$ & 0 & 0 & 0 & 0 & 0 \\
\hline $\mathrm{BOOK}$ & $(858)$ & (589) & (355) & $(166)$ & 51 & 1,741 & 2,024 & 2,347 & 2,715 & 3,132 \\
\hline TAXABLE & (858) & $(589)$ & (355) & (166) & 51 & 1,741 & 2,024 & 2,347 & 2,715 & 3,132 \\
\hline TAX (-PAY, + CREDIT) & 429 & 294 & 178 & 83 & $(25)$ & $(870)$ & $(1,012)$ & $(1,173)$ & $(1,357)$ & $(1,566)$ \\
\hline & & & & & & & & & & $-\quad \ldots$ \\
\hline $\mathrm{BOOK}$ & $(858)$ & $(589)$ & (355) & $(166)$ & 51 & 1,741 & 2,024 & 2,347 & 2,715 & 3,132 \\
\hline $\operatorname{TAX}$ & & & & & & & & & & \\
\hline DEPRECIATION & 1.030 & 1,511 & 1,442 & 1,442 & 1,442 & $\overline{0}$ & $\mathbf{0}$ & $\overline{0}$ & $\mathbf{0}$ & $\mathbf{0}$ \\
\hline INVESTMENT & $(6,866)$ & 0 & 0 & 0 & 0 & $\overline{0}$ & $\mathbf{0}$ & $\overline{0}$ & $\overline{0}$ & $\overline{0}$ \\
\hline TAX CREDIT (ITC,ETC) & 1,717 & 0 & 0 & 0 & 0 & 0 & 0 & 0 & 0 & 0 \\
\hline CASH FLOW & $(4,549)$ & 1,216 & 1,264 & 1,359 & 1,467 & 870 & 1,012 & 1,173 & 1,357 & 1,566 \\
\hline R.O.I (D.C.F) = & 23.159 & & & & & & & & & \\
\hline R.0.I (D.C.F) $=$ & $23.15 z$ & & & & & & & & & \\
\hline
\end{tabular}

PART II STUDY - SINGLE UNIT GRAVITY HEAD WITH $38.9408 \mathrm{c} / 1000$ lb BRINE COST - WELL COST EXCLUDED 


\begin{tabular}{|c|c|c|c|c|c|c|c|c|c|c|}
\hline YEAR & 1 & 2 & 3 & 4 & 5 & 6 & 7 & 8 & 9 & 10 \\
\hline POWER SALE & 1,016 & 2,776 & 3,082 & 3,421 & 3,797 & 4,215 & 4,678 & 5,193 & 5,764 & 6,398 \\
\hline CAPACITY PAYMENTS & 51 & 203 & 203 & 203 & 203 & 203 & 203 & 203 & 203 & 203 \\
\hline BRINE COSTS & $(531)$ & $(1,391)$ & $(1,482)$ & $(1,578)$ & $(1,681)$ & $(1,790)$ & $(1,906)$ & $(2,030)$ & $(2,162)$ & $(2,302)$ \\
\hline $0 \& M$ COSTS & $(550)$ & $(1,210)$ & $(1,331)$ & $(1,464)$ & $(1,611)$ & $(1,772)$ & $(1,949)$ & $(2,144)$ & $(2,358)$ & $(2,594)$ \\
\hline DEPRECIATION & $(1,047)$ & $(1.536)$ & $(1,466)$ & $(1,466)$ & $(1,466)$ & 0 & 0 & 0 & 0 & 0 \\
\hline BOOK & $(1,061)$ & $(1,158)$ & $(994)$ & $(885)$ & $(757)$ & 857 & 1,027 & 1,223 & 1,447 & 1,705 \\
\hline & & & & & & & & & & \\
\hline TAX (-PAY, + CREDIT) & 531 & 579 & 497 & 442 & 379 & $(428)$ & (513) & $(611)$ & $(724)$ & (853) \\
\hline & & & & & & & & & & \\
\hline$\frac{\text { BOOK }}{\text { TAX }}$ & $(1,061)$ & $(1,158)$ & (994) & $(885)$ & $(757)$ & 857 & 1,027 & 1,223 & 1,447 & 1,705 \\
\hline DAXPRECIATION & 1007 & 7526 & & & & & & & & \\
\hline$\frac{\text { DEPRECIATION }}{\text { INVESTMENT }}$ & $\frac{1.047}{16}$ & 1,536 & 1,466 & 1,466 & 1,466 & o & $\mathbf{0}$ & $\mathbf{0}$ & $\mathbf{0}$ & $\mathbf{0}$ \\
\hline $\begin{array}{l}\text { INVESTMENT } \\
\text { TAX CREDIT (ITC,ETC) }\end{array}$ & $\frac{(6,983)}{7}$ & $\frac{0}{n}$ & 0 & 0 & 0 & 0 & $\mathbf{0}$ & $\mathbf{0}$ & 0 & $\mathbf{0}$ \\
\hline CASH FLOW & $\frac{1,746}{(4,720)}$ & $\frac{0}{957}$ & 0 & 0 & 0 & 0 & 0 & 0 & 0 & 0 \\
\hline & & & & & & & & & & \\
\hline R.O.I (D.C.F) = & 10.28 & & & & & & & & & \\
\hline
\end{tabular}

PART II STUDY - SINGLE UNIT BINARY WITH 30.2873c/1000 1b. BRINE COST-WELL COST EXCLUDED. 


\begin{tabular}{|c|c|c|c|c|c|c|c|c|c|c|}
\hline YEAR & 1 & 2 & 3 & 4 & 5 & 6 & 7 & $\overline{8}$ & 9 & 10 \\
\hline POWER SALE & 1,016 & 2,776 & 3,082 & 3,421 & 3,797 & 4,215 & 4,678 & 5,193 & 5,764 & 6,398 \\
\hline CAPACITY PAYMENTS & 51 & 203 & 203 & 203 & 203 & 203 & 203 & 203 & 203 & 203 \\
\hline BRINE COSTS & $(682)$ & $(1,789)$ & $(1,905)$ & $(2,029)$ & $(2,161)$ & $(2,301)$ & $(2,451)$ & $(2,610)$ & $(2,780)$ & $(2,960)$ \\
\hline O \& M COSTS & $(550)$ & $(1,210)$ & $(1,331)$ & $(1,464)$ & $(1,611)$ & $(1,772)$ & $(1,949)$ & $(2,144)$ & $(2,358)$ & $\frac{10,000)}{(2,594)}$ \\
\hline DEPRECIATION & $(1,047)$ & $(1,536)$ & $(1,466)$ & $(1,466)$ & $(1,466)$ & 0 & 0 & 0 & 0 & 0 \\
\hline $\mathrm{BOOK}$ & $(1,213)$ & $(1,555)$ & $(1,417)$ & $(1,335)$ & $(1,237)$ & 345 & 482 & 643 & 830 & 1,047 \\
\hline TAXABLE & $(1,213)$ & $(1,555)$ & $(1,417)$ & $(1,355)$ & $(1.237)$ & 345 & 482 & 643 & 830 & 1.047 \\
\hline TAX $(-\mathrm{PAY},+$ CREDIT $)$ & 606 & $\frac{12.07}{778}$ & 709 & $\frac{12,568}{668}$ & 619 & $(173)$ & $\frac{402}{(241)}$ & $\frac{045}{(321)}$ & $\frac{850}{(415)}$ & $\frac{1,047}{(524)}$ \\
\hline BOOK & $(1,213)$ & $(1,555)$ & $(1,417)$ & $(1,335)$ & $(1.237)$ & 345 & 482 & 643 & 830 & 1,047 \\
\hline $\operatorname{TAX}$ & & & & & & & & & & \\
\hline DEPRECIATION & 1,047 & 1,536 & 1,466 & 1,466 & 1,466 & 0 & $\mathbf{0}$ & 0 & 0 & $\mathbf{0}$ \\
\hline INVESTMENT & $(6,983)$ & 0 & 0 & 0 & 0 & 0 & 0 & 0 & 0 & 0 \\
\hline TAX CREDIT (ITC,ETC) & 1,746 & 0 & 0 & 0 & 0 & 0 & 0 & 0 & 0 & 0 \\
\hline CASH FLOW & $(4,796)$ & 759 & 758 & 799 & 848 & 173 & 241 & 321 & 415 & 524 \\
\hline R.O.I (D.C.F) $=$ & 0.28 & & & & & & & & & \\
\hline
\end{tabular}

PART II STUDY - SINGLE UNIT BINARY WITH 38.9408c/1000 lb BRINE COST - WELLS EXCLUDED 


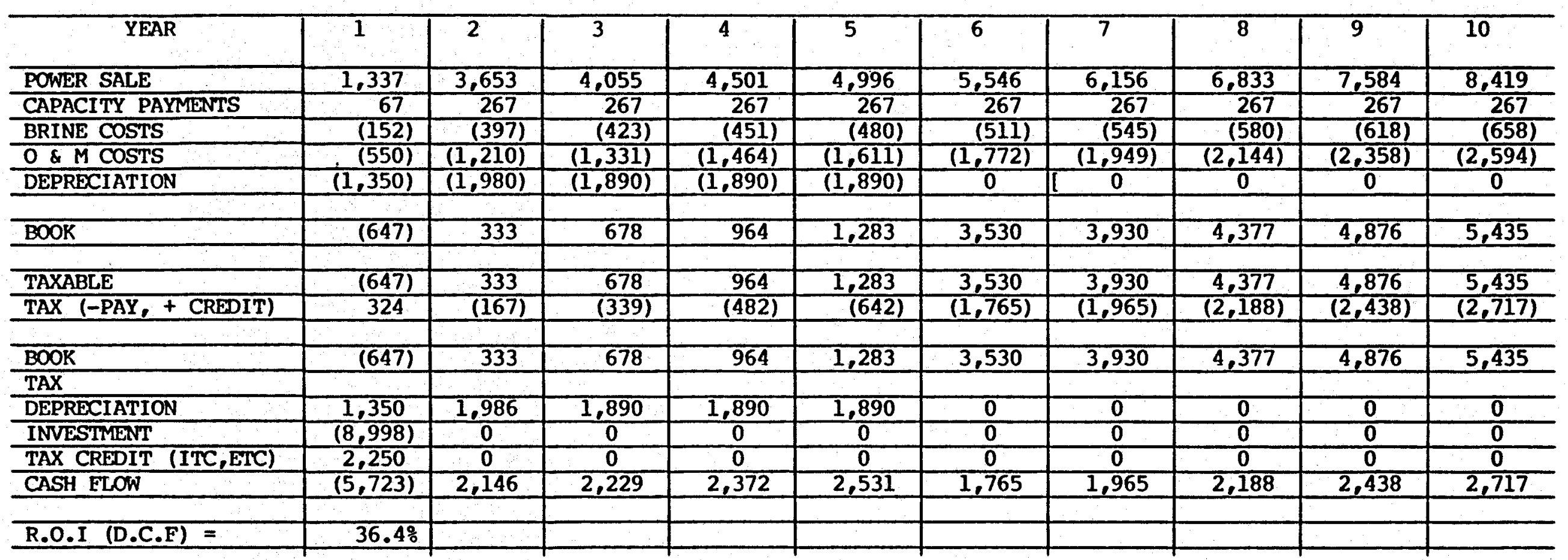

PART II STUDY - SINGLE UNIT GRAVITY HEAD WITH 8.6535c/1000 1b BRINE COST - WELL COST INCLUDED 


\begin{tabular}{|c|c|c|c|c|c|c|c|c|c|c|}
\hline YEAR & 1 & 2 & 3 & 4 & 5 & 6 & 7 & 8 & 9 & 10 \\
\hline POWER SALE & 1,337 & 3,653 & 4.055 & 4,501 & 4,996 & 5,546 & 6,156 & 6,833 & 7,584 & 8,419 \\
\hline CAPACITY PAYMENTS & 67 & 267 & 267 & 267 & 267 & 267 & 267 & 267 & 267 & 267 \\
\hline BRINE COSTS & (379) & $(994)$ & $(1,058)$ & $(1,127)$ & $(1,200)$ & $(1,278)$ & $(1,361)$ & $(1,450)$ & $(1.544)$ & $(1,644)$ \\
\hline O\& M COSTS & $(550)$ & $(1,210)$ & $(1,331)$ & $(1,464)$ & $(1,611)$ & $(1,772)$ & $(1,848)$ & $(2,144)$ & $(2,358)$ & $(2,594)$ \\
\hline DEPRECIATION & $(1,030)$ & $(1,511)$ & $(1,442)$ & $(1,442)$ & $(1,442)$ & 0 & 0 & 0 & 0 & \\
\hline BOOK & (555) & 206 & 491 & 735 & 1,011 & 2,763 & 3,113 & 3,507 & 3,950 & 4,448 \\
\hline TAXABLE & (555) & 206 & 491 & 735 & 1,011 & 2,763 & 3,113 & 3,507 & 3,950 & 4,448 \\
\hline TAX (-PAY, + CREDIT) & 278 & $(103)$ & (246) & (368) & $(505)$ & $(1,382)$ & $(1,557)$ & $(1,753)$ & $(1,975)$ & $(2,224)$ \\
\hline $\mathrm{BOOK}$ & (555) & 206 & 491 & 735 & 1,011 & 2,763 & 3,113 & 3,507 & 3,950 & 4,448 \\
\hline TAX & & & & & & & & & & \\
\hline DEPRECIATION & 1,030 & 1,511 & 1,442 & 1,442 & 1,442 & $\overline{0}$ & $\overline{\mathbf{0}}$ & $\mathbf{0}$ & $\overline{0}$ & $\overline{0}$ \\
\hline INVESTMENT & $(6,866)$ & 0 & 0 & 0 & 0 & 0 & 0 & 0 & $\mathbf{0}$ & 0 \\
\hline TAX CREDIT (ITC,ETC) & 1,717 & 0 & 0 & 0 & 0 & 0 & 0 & 0 & 0 & 0 \\
\hline CASH FLOW & $(4.397)$ & 1,614 & 1,688 & 1,810 & 1,957 & 1,382 & 1,557 & 1,753 & 1,975 & 2,224 \\
\hline R.O.I (D.C.F $)=$ & $36.4 \%$ & & & & & & & & & \\
\hline
\end{tabular}

PART II STUDY - SINGLE-UNIT GRAVITY HEAD WITH $21.6338 \mathrm{c} / 1000$ 1b BRINE COST - WELL COST INCLUDED 


\begin{tabular}{|c|c|c|c|c|c|c|c|c|c|c|}
\hline YEAR & 1 & 2 & 3 & 4 & 5 & 6 & 7 & 8 & 9 & 10 \\
\hline POWER SALE & 1,016 & 2,776 & 3,082 & 3,421 & 3,797 & 4,215 & 4,678 & 5,193 & 5,764 & 6,398 \\
\hline CAPACITY PAYMENTS & 51 & 203 & 203 & 203 & 203 & 203 & 203 & 203 & 203 & 203 \\
\hline BRINE COSTS & (152) & (397) & $(423)$ & (451) & $(480)$ & $(511)$ & (545) & $(580)$ & $(618)$ & $(658)$ \\
\hline O\&M COSTS & $(550)$ & $(1,210)$ & $(1,331)$ & $(1,464)$ & $(1,611)$ & $(1,772)$ & $(1,949)$ & $(2,144)$ & $(2,358)$ & $(2,594)$ \\
\hline DEPRECIATION & $(1,369)$ & $(2,008)$ & $(1,916)$ & $(1,916)$ & $(1,916)$ & 0 & 0 & 0 & 0 & 0 \\
\hline BOOK & $(1,004)$ & $(636)$ & $(386)$ & $(207)$ & (7) & 2,135 & 2,388 & 2,673 & 2,992 & 3,350 \\
\hline TAXABLE & $(1,004)$ & $(636)$ & $(386)$ & $(207)$ & (7) & 2,135 & 2,388 & 2,673 & 2,992 & 3,350 \\
\hline TAX (-PAY, + CREOIT) & 502 & 318 & 193 & 104 & 3 & $(1,067)$ & $(1,194)$ & $(1,336)$ & $(1,496)$ & $(1,675)$ \\
\hline BOOK & $(1,004)$ & $(636)$ & $(386)$ & (207) & (7) & 2,135 & 2,388 & 2,673 & 2,992 & 3,550 \\
\hline TAX & & & & & & & & & & \\
\hline DEPRECIATION & 1,369 & 2,008 & 1,916 & 1,916 & 1,916 & $\overline{0}$ & $\overline{0}$ & $\overline{0}$ & $\overline{0}$ & $\overline{0}$ \\
\hline INVESTMENT & $(9,126)$ & 0 & 0 & 0 & 0 & 0 & 0 & 0 & 0 & 0 \\
\hline TAX CREDIT (ITC,ETC) & 2,281 & 0 & 0 & 0 & $\mathbf{0}$ & 0 & 0 & 0 & 0 & 0 \\
\hline CASH FLOW & $(5,977)$ & 1,690 & 1,723 & 1,813 & 1,913 & 1,067 & 1,194 & 1,336 & 1,496 & 1,675 \\
\hline R.O.I (D.C.F) $=$ & $22.6 \%$ & & & & & & & & & \\
\hline
\end{tabular}

PART II STUDY - SINGLE UNIT BINARY WITH 8.6535c/1000 lb - BRINE COST - WELL COST INCLUDED 


\begin{tabular}{|c|c|c|c|c|c|c|c|c|c|c|}
\hline YEAR & 1 & 2 & 3 & 4 & 5 & 6 & 7 & 8 & $\overline{9}$ & 10 \\
\hline POWER SALE & 1,016 & 2,776 & 3,082 & 3,421 & 3,797 & 4,215 & 4,678 & 5,193 & 5,764 & 6,938 \\
\hline CAPACITY PAYMENTS & 51 & 203 & 203 & 203 & 203 & 203 & 203 & 203 & 203 & 203 \\
\hline BRINE COSTS & (379) & (994) & $(1,058)$ & $(1,127)$ & $(1,200)$ & $(1,278)$ & $(1,362)$ & $(1,450)$ & $(1,544)$ & $(1,645)$ \\
\hline $0 \& M$ COSTS & $(550)$ & $(1,210)$ & $(1,331)$ & $(1,464)$ & $(1,611)$ & $(1,772)$ & $(1,949)$ & $(2,144)$ & $(2,358)$ & $(2,594)$ \\
\hline DEPRECIATION & $(1,047)$ & $(1,536)$ & $(1,466)$ & $(1,466)$ & $(1,466)$ & 0 & 0 & 0 & 0 & 0 \\
\hline$\overline{B O O K}$ & $(910)$ & $(760)$ & (571) & $(434)$ & (277) & 1,368 & 1,571 & 1,803 & 2,065 & 2,363 \\
\hline TAX (-PAY, + CREDIT) & 455 & 380 & 285 & 217 & 139 & $(684)$ & $(786)$ & $(901)$ & $(1,033)$ & $(1,182)$ \\
\hline$\overline{B O O K}$ & $(910)$ & $(760)$ & $(571)$ & $(434)$ & $(277)$ & 1.368 & 1.571 & 1.803 & 2.065 & 2.363 \\
\hline $\operatorname{TAX}$ & & & & & & 1,200 & & 1,002 & 2,005 & 2,003 \\
\hline DEPRECIATION & 1,047 & 1,536 & 1,466 & 1,466 & 1,466 & $\overline{0}$ & 0 & $\overline{0}$ & 0 & 0 \\
\hline INVESTMENT & $(6,938)$ & 0 & 0 & 0 & 0 & 0 & $\mathbf{0}$ & 0 & 0 & 0 \\
\hline TAX CREDIT (ITC,ETC) & 1,746 & 0 & 0 & 0 & 0 & 0 & 0 & 0 & 0 & 0 \\
\hline CASH FLOW & $(4,644)$ & 1,156 & 1,181 & 1,250 & 1,328 & 684 & 786 & 901 & 1,033 & 1,182 \\
\hline R.O.I (D.C.F) $=$ & 18.39 & & & & & & & & & \\
\hline
\end{tabular}

PART II STUDY - SINGLE-UNIT BINARY WITH $21.6338 \mathrm{c} / 1000$ lb BRINE COST - WELL COST INCLUDED 


\begin{tabular}{|c|c|c|c|c|c|c|c|c|c|c|}
\hline YEAR & 1 & 2 & 3 & 4 & 5 & 6 & 7 & 8 & 9 & 10 \\
\hline POWER SALE & 0 & 5,639 & 15,409 & 17,104 & 18,985 & 21.073 & 23,391 & 25,965 & 28,821 & 31,991 \\
\hline CAPACITY PAYMENTS & 0 & 316 & 1,265 & 1.265 & 1,265 & 1,265 & 1,265 & 1,265 & 1,265 & 1,265 \\
\hline BRINE COSTS & 0 & $(1,615)$ & $(4,233)$ & $(4,508)$ & $(4,802)$ & $(5,114)$ & $(5,446)$ & $(5,800)$ & $(6,177)$ & $(6,579)$ \\
\hline O\& M COSTS & 0 & $(1,210)$ & $(2,662)$ & $(2,928)$ & $(3,221)$ & $(3,543)$ & $(3,897)$ & $(4,287)$ & $(4,716)$ & $(5,187)$ \\
\hline DEPRECIATION & $(964)$ & $(7,517)$ & $(10,301)$ & $(9,894)$ & $(9,894)$ & $(8,544)$ & 0 & 0 & 0 & 0 \\
\hline BOOK & (964) & $(4,386)$ & (522) & 1,038 & 2,334 & 5,138 & 15,313 & 17.142 & 19,193 & 21,490 \\
\hline TAXABLE & (964) & $(4,386)$ & (522) & 1,038 & 2,334 & 5,138 & 15,313 & 17,142 & 19,193 & 21,490 \\
\hline TAX (-PAY, + CREDIT) & 482 & 2,193 & 261 & (519) & $(1,167)$ & $(2,569)$ & $(7,656)$ & $(8,571)$ & $(9.596)$ & $(10,745)$ \\
\hline $\mathrm{BOOK}$ & (964) & $(4,386)$ & (522) & 1,038 & 2,334 & 5,138 & 15,313 & 17,142 & 19,193 & 21,490 \\
\hline TAX & & & & & & & & & & \\
\hline DEPRECIATION & 964 & 7,517 & 10,301 & 9,894 & 9,894 & 8,544 & 0 & $\mathbf{0}$ & $\mathbf{0}$ & $\mathbf{0}$ \\
\hline INVESTMENT & $(30,776)$ & $(16,337)$ & 0 & 0 & 0 & 0 & 0 & $\overline{0}$ & $\mathbf{0}$ & 0 \\
\hline TAX CREDIT (ITC,ETC) & 7,694 & 4,084 & 0 & 0 & 0 & 0 & 0 & $\overline{0}$ & $\overline{0}$ & 0 \\
\hline CASH FLOW & $(22,600)$ & $(6,929)$ & 10,039 & 10,413 & 11,061 & 11,113 & 7,656 & 8,571 & 9,596 & 10,745 \\
\hline R.O.I (D.C.F) $=$ & 23.528 & & & & & & & & & \\
\hline
\end{tabular}

PART II STUDY - 19 mw BINARY GATHERING PLANT WITH 17.247c/1000 lb BRINE COST - WELLS INCLUDED 